\title{
NETWORK FLOW ORIENTED APPROACHES FOR VEHICLE SHARING RELOCATION PROBLEMS
}

\author{
Alain Quilliot ${ }^{1 *}$, Samuel Deleplanque ${ }^{2}$, \\ Antoine Sarbinowski ${ }^{3}$ and Annegret Wagler ${ }^{4}$
}

Received April 24, 2017 / Accepted November 30, 2017

\begin{abstract}
Managing a one-way vehicle sharing system means periodically moving free access vehicles from excess to deficit stations in order to avoid local shortages. We propose and study here several network flow oriented models and algorithms which deal with a static version of this problem while unifying preemption and non preemption as well as carrier riding cost, vehicle riding time and carrier number minimization. Those network flow models are vehicle driven, which means that they focus on the way vehicles are exchanged between excess and deficit stations. We perform a lower bound and approximation analysis which leads us to the design and test of several heuristics. One of them involves implicit dynamic network handling.
\end{abstract}

Keywords: Network Flow, routing, Vehicle sharing.

\section{INTRODUCTION}

Vehicle Sharing systems [14, 20, 28, 29] are emerging mobility systems which aim at compromising between purely individual mobility and rather rigid public transportation. Such a system is composed of a set of stations, at which free access vehicles are parked. Those vehicles may be bicycles or electric cars. There exists a special station called Depot, in which a set of carriers (trucks, self-platoon convoys, ...) are waiting: they periodically exchange vehicles between the stations and eventually provide them with additional vehicles. A trend is to make the system be a one-way system: users are not imposed to give vehicles back at the stations where they picked them up. This feature makes the system more attractive, but also raises the eventuality of unbalanced situations: stations may become overfilled other under-filled, provoking local shortages or making users unable to give their vehicles back. This makes arise two decision problems:

\footnotetext{
*Corresponding author.

${ }^{1}$ LIMOS CNRS 6158, Labex IMOBS3, UCA Clermont-Ferrand, France. E-mail: alain.quilliot@isima.fr

${ }^{2}$ ULB, Bruxelles, Belgium. E-mail: deleplanque.samuel@ gmail.com

${ }^{3}$ Labex IMOBS3, UCA Clermont-Ferrand, France. E-mail: antoine.sarbinowski@isima.fr

${ }^{4}$ LIMOS CNRS, UCA Clermont-Ferrand, France. E-mail: awagler@isima.fr
} 
- a strategic level problem [8, 9, 14, 25, 29], about the way stations are located and capacitated and about the pricing of the system [29]. One must simultaneously maximize some global Access Demand, and minimize costs which involve not only infrastructure costs but also running costs related to periodical vehicle relocation. Though this Vehicle Sharing Station Location (VSSL) problem looks like a standard Facility Location problem $[13,19,22,26,30]$, addressing it is difficult in practice, since estimating the way Access Demand depends on the way stations are located can only be done through rough approximation.

- an operational (or tactical) level problem (see [5, 6, 7, 10, 11, 18, 20, 21, 23, 24, 25, 27]), about the way vehicles are periodically moved from excess to deficit stations in order rebalance the system (Relocation Process). Performing this process while meeting both economic and quality of service purposes means addressing a Vehicle Sharing Relocation problem (VSR).

This contribution is devoted to the operational level, that means to the VSR Problem, which also appears as a slave sub-problem in any bi-level VSSL formulation. Related VSR models may be:

- static: at some time during the process, excess and deficit stations are identified, together with excess and deficit amount of vehicles. One must make the carriers move vehicles from excess to deficit stations, while minimizing some operational cost, function of the vehicle riding time, of the number of carriers and of the carrier riding time, while keeping the total duration of the process from exceeding a makespan threshold;

- dynamic: one knows, for every station $x$, at which time vehicles are going to be demanded or given back by the users. Then one schedules the carriers in order to meet most demands and avoid any unbalanced situation, while minimizing some operational cost;

- on line: the context is the same as in the dynamic case, but knowledge about demands is incomplete and uncertain.

Preemption may be allowed: a carrier may load some vehicle at some station and drop it at another station, before some other (or eventually the same) carrier comes, loads it again and brings it until a third station.

In practice, VSR models have to be handled on line [4, 18, 20, 23]: relocation is performed in a continuous way and, at any time, knowledge about customer requests is incomplete and uncertain. Still, as it is usual when it comes to scheduling or routing decisional problems, it is appropriated, in order to better understand the problem and design efficient strategies, to first deal with a static or eventually a dynamic version. VSR literature makes appear several static models (see $[3,4,10]$ ) which have been addressed through metaheuristic schemes or through hierarchical decomposition into a routing master model, handled through local search, and a load/unload network flow slave model [1]. Most authors impose restrictions on the number of carriers and the components of the cost function $[5,6,7,10]$, most often reduced to the carrier riding time. 
Some authors consider time indexed requests $[11,27]$ and address the resulting model through a Benders decomposition scheme. None of them links non preemption and preemption, while, even if not practical from the point of view of a central manager, preemption may be used as a relaxation of non preemption and help into designing algorithms.

More, one may notice that a common feature of the above mentioned static and dynamic models is that they are carrier oriented [24, 25], in the sense that they focus on the construction of the recollection tours which are run by the carriers, and consider the routing of the vehicles inside the carriers as a kind of slave object [10]. Such an approach may be criticized because of the lack of a backward link between the master carrier tour collection and the vehicle sub-problem: the search for the master carrier tour collection is then performed in a somewhat blind way (genetic algorithms, ...).

So we adopt here the opposite point of view and consider that performing a relocation process means routing vehicles from excess stations to deficit ones in a way which make them share, as often as possible, related carriers. This leads us to propose models which stress the role played by the vehicle network flow induced by the relocation process, and then derive alternative approaches to carrier driven ones, which we say to be vehicle driven: the vehicle routing strategy becomes the master object, which determines in turn the carrier routes. This allows us to link preemptive and non preemptive VSR models and point out that understanding preemption as a relaxation of non preemption leads us to a common Network Flow framework.

The paper is organized as follows. We first provide (Section 2) a general framework for both preemptive and non preemptive static VSR, which mixes several performance criteria: economic cost of the relocation process (carrier number and carrier riding cost), and quality of service (unavailability of the vehicles during the process). We reformulate resulting models as Network Flow models, making appear preemption as a relaxation of non preemption. We keep on (Section 3) by performing a lower bound analysis of this VSR model. In Section 4, we propose a first heuristic scheme, which considers the way vehicles are distributed from excess stations to deficit ones as the master object of a Min Cost Assignment/Pick up and Delivery hierarchical decomposition scheme, and state an approximation result for what we call the min-cost assignment strategy. In Section 5 and 6, we propose and test heuristics, which deal with aggregated vehicle and carrier flow vectors and turn them into solutions of respectively Non Preemptive and Preemptive VSR. One of those heuristics involves the implicit management of large size dynamic network.

\section{VSR PREEMPTIVE AND NON PREEMPTIVE CARRIER ORIENTED MODELS}

VSR (Vehicle Sharing Relocation Problem) Instances: We consider here a set $X$ of stations, one of them being a specific station Depot. Any station $x$ is provided with a coefficient $v(x)$, which tells us that $v(x)$ vehicles are in excess at station $x$ : if $v(x)$ is strictly negative, then carriers need to bring $-v(x)$ vehicles to station $x$ ( $x$ is then said to be a deficit station); if $v(x)$ is strictly positive, then $x$ is an excess station and carriers have to remove $v(x)$ vehicles from $x$; 
if $v(x)=0$ then $x$ is said to be neutral. Carriers are initially located at Depot and they all have a same capacity $C A P$. We suppose that $\Sigma_{x \in X} v(x)=0$, which means that some stations may be used to bring additional vehicles to the system, or, conversely, to remove some of them. We also suppose that Depot is neutral. Any station $x$ is provided with a capacity $C(x)$. DIST denotes the X.X time matrix: DIST $T_{x, y}$ is the time required for a carrier to go from station $x$ to station $y$. T-Max is the maximal makespan of the relocation process: the total time for this process cannot exceed T-Max. By the same way, COST denotes the X.X carrier cost matrix: $\operatorname{COST}_{x, y}$ is the integrated cost (energy, human resource, ...) induced by a move of a carrier from station $x$ to station $y$, when this move is performed in DIST $x, y$ time units. Both matrices DIST and COST satisfy the Triangle Inequality and are such that $\operatorname{COST}_{x, x}=D I S T_{x, x}=0$ for any station $x$. IdleCost denotes the waiting cost induced for a carrier when it remains at any station $x \neq$ Depot during one time unit. We suppose (Extended Cost Hypothesis) that if a carrier moves from $x$ to $y$ at a reduced speed in time $t \geq D I S T_{x, y}$, then the induced extended $\cos E$-COST $T_{x, y, t}$ is equal to $\operatorname{COST}_{x, y}+I d l e-C o s t .\left(t-D I S T_{x, y}\right)$. All this defines a VSR instance (X,v,C,CAP, T-Max, DIST, COST).

\subsection{Non Preemptive VSR Model}

A VSR tour $\Gamma$ is a finite sequence $\Gamma_{\text {Route }}=\left\{x_{0}=\right.$ Depot, $x_{1}, \ldots, x_{n(\Gamma)}=$ Depot $\}$ of stations, which is called a route, given together with a loading strategy, that means with 2 sequences $\Gamma_{\text {Load }}=\left\{L_{0}=0, L_{1}, \ldots, L_{n(\Gamma)}\right\}$ and $\Gamma_{\text {Time }}=\left\{T_{0} \geq 0, T_{1}, \ldots, T_{n(\Gamma)}\right\}$ of coefficients whose meaning is: a carrier which follows the route $\Gamma_{\text {Route }}$ loads, at time $T_{i}, L_{i}$ vehicles at station $x_{i}$ (unloads in case $\left.L_{i}<0\right)$. The length of $\Gamma_{\text {Route }}$ in the COST sense is given by $L-\operatorname{COST}\left(\Gamma_{\text {Route }}\right)$ $=\Sigma_{j} C O S T_{x j, x_{j}+1}$. The length of $\Gamma_{\text {Route }}$ in the DIST sense is given by $\operatorname{L-DIST}\left(\Gamma_{\text {Route }}\right)=\Sigma_{j}$ $\operatorname{DIST}_{x j, x j+1}$. The cost $L-E-\operatorname{COST}(\Gamma)$ of $\Gamma$ is given by: $L-E-\operatorname{COST}(\Gamma)=L-\operatorname{COST}\left(\Gamma_{\text {Route }}\right)+$ Idle$\operatorname{Cost} .\left(T_{n(\Gamma)}-T_{0}\right)$. For any $i$, we denote by $L_{i}^{*}=\Sigma_{j=0 . . i} L j$ the load of the carrier when it leaves station $x_{i}$.

This VSR tour $\Gamma$ is Non Preemptive VSR feasible if:

- For any $i=0, \ldots, n(\Gamma)-1, T-M a x \geq T_{i+1} \geq T_{i}+D I S T_{x i, x i+1}$;

- For any $i=0, \ldots, n(\Gamma)-1,0 \leq L_{i}^{*}=\Sigma_{j=0 \ldots i} L j \leq C A P$;

- $\Sigma_{j=0 \ldots n(\Gamma)} L_{j}=0$;

- For any $j$ such that $v\left(x_{j}\right) \geq 0\left(v\left(x_{j}\right) \leq 0\right)$, then $v\left(x_{j}\right) \geq L j \geq 0\left(v\left(x_{j}\right) \leq L j \leq 0\right)$.

Explanation: (E1): A carrier needs at least DIST $T_{x i, x i+1}$ time units to go from $x_{i}$ to $x_{i+1}$; (E2, E3): Current carrier load $L_{i}^{*}$ cannot exceed the capacity $C A P$, and this load is null at the end of the tour; (E4): loading (unloading) operations are respectively restricted to excess (deficit) stations, which means that we impose a given vehicle to be moved from an origin station to a destination station by exactly one carrier (Non Preemption). 
Given scaling coefficients $\alpha, \beta, \delta$ together with a VSR instance $(X, v, C, C A P, T$-Max, DIST, COST), we set:

Non Preemptive VSR Model: \{Compute a VSR feasible tour collection $\Gamma^{*}=(\Gamma(k)$, $k=1 \ldots K)$ such that:

- For any station $x: \Sigma_{k} \Sigma_{i}$ such that $x(k) i={ }_{x} L(k)_{i}=v(x)$.

- Minimize Global-Cost $\left(\Gamma^{*}\right)=\alpha . K+\beta . \Sigma_{k} L-E-\operatorname{COST}(\Gamma(k))+$ $\delta .\left(\Sigma_{k} \Sigma_{j}\left(D I S T_{x(k) j, x(k) j+1} \cdot L^{*}(k)_{j}\right)\right\}$.

Explanation: (E5): For any excess station $x, v(x)$ vehicles have to be picked up in $x$, and for any deficit station $x,-v(x)$ vehicles have to be delivered to $x$. Global-Cost $\left(\Gamma^{*}\right)$ is a weighted sum of the carrier number, the carrier riding cost, and the vehicle riding time (time vehicles spend into the carriers).

Remark 1. Because of non preemption, every move from $x(k)_{i}$ to $x(k)_{i+1}$ in $\Gamma(k)$ may be performed in exactly $D I S T_{x(k) i x(k) i+1}$ time units; Thus $\Sigma_{k} L-E-\operatorname{COST}(\Gamma(k))$ may be replaced by $\Sigma_{k} L-\operatorname{COST}\left(\Gamma(k)_{\text {Route }}\right.$. By the same way, any neutral stations but the Depot station may be removed from the input of the Non Preemptive VSR model.

Remark 2 (About MIP Models and Complexity). Modeling VSR through a MIP (Mixed Integer Linear Program) is possible, but inefficient. The reason is that there is no a priori bound about the number of times a given station is going to be visited by a same carrier. As for complexity, in the case when $K=1$ ( $\alpha$ very large), $v(x)$ values are equal to 1 or $-1, C A P=1$ and $\delta=0$, our problem is equivalent to the Travelling Salesman Problem set on a bipartite graph (the excess stations on one side and the deficit ones on the other side), which is NP-Hard. Non Preemptive VSR also contains the Uncapacitated Swapping Problem, which is also NP-Hard (see [1]).

\subsection{Loading Strategy Flow Model Related to a VSR Route Collection $\Gamma_{\text {Route }}^{*}$}

Let us suppose now that we are provided with a collection $\Gamma_{\text {Route }}^{*}=\left\{\Gamma_{\text {Route }}(1), \ldots, \Gamma_{\text {Route }}(K)\right\}$ of $K$ carrier routes, all with length $\leq T$-Max. Following [10], we define a network $H\left(\Gamma_{\text {Route }}\right)$ as follows (see Fig. 1):

- Nodes of $H\left(\Gamma_{\text {Route }}^{*}\right)$ are:

$\circ$ copies of the nodes $x(k)_{j}$ of $\Gamma_{\text {Route }}(1), \ldots, \Gamma_{\text {Route }}(K)$ considered as being all distinct;

○ a source $s$ and a sink $p$;

○ nodes $\operatorname{Exc}(x), x \in X$, excess nodes;

○ nodes $\operatorname{Def}(x), x \in X$, deficita nodes. 
- Arcs $e$ of $H\left(\Gamma_{\text {Route }}\right)$ and related costs $C_{e}$ are:

$\circ$ Route arcs $e=\left(x(k)_{j}, x(k)_{j+1}\right)$ of the routes $\Gamma_{\text {Route }}(k)$, with cost $C_{e}=$ $D I S T_{x(k) j, x(k) j+1}$;

- Excess $\operatorname{arcs} e=\left(\operatorname{Exc}(x), x(k)_{j}\right), x \in X, x$ excess, such that the image in $X$ of $x(k)_{j}$ is $x$, with $C_{e}=0$;

○ Deficit arcs $e=\left(y(k)_{j}, \operatorname{Def}(y)\right), y$ deficit, such that $y(k)_{j}$ is $y$, with $C_{e}=0$;

- Input arcs $e=(s, \operatorname{Exc}(x)), x$ excess, and output $\operatorname{arcs} e=(\operatorname{Def}(y), p), y$ deficit, with $C_{e}=0$.

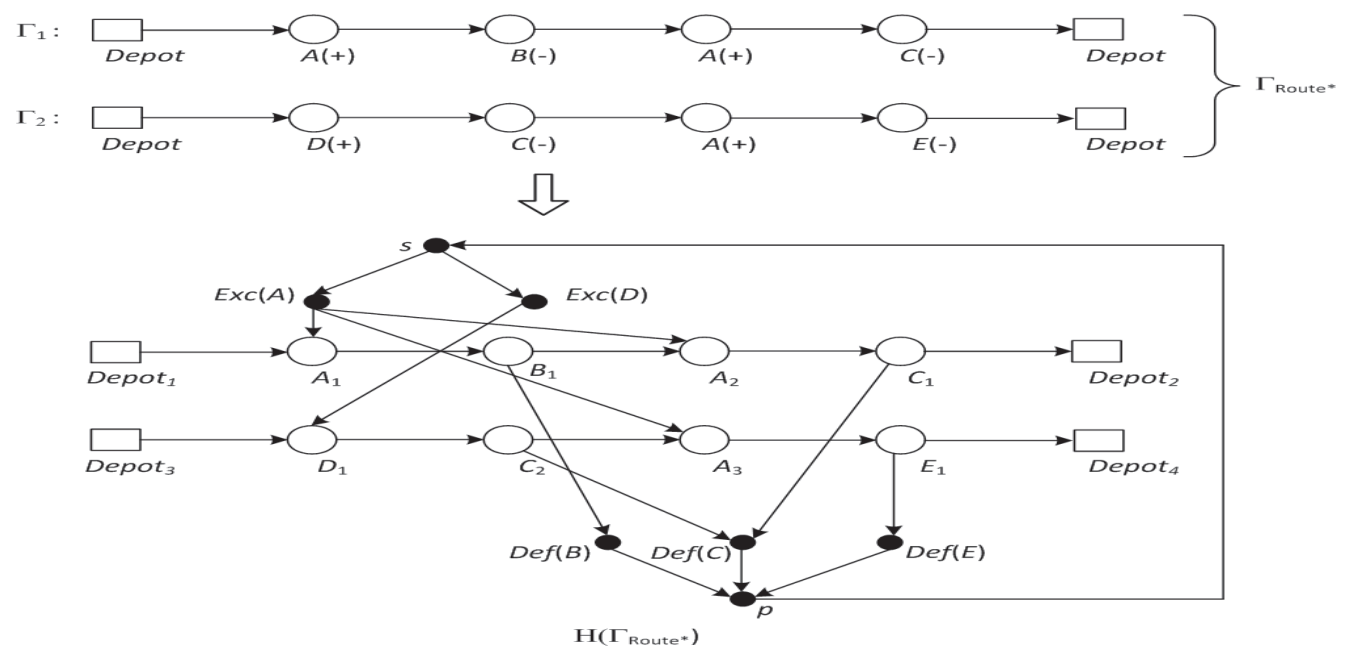

Figure 1

Then we may set:

Load-NP-VSR Model: $\left\{\right.$ Compute on $H\left(\Gamma_{\text {Route }}^{*}\right)$ a non negative integral arc indexed flow vector Z such that:

- for any route arc e, $Z_{e} \leq C A P$;

- for any input arc $e=(s, \operatorname{Exc}(x))$, xexcess,$Z_{e}=v(x)$;

$\circ$ for any output arc $e=(\operatorname{Def}(y), p)$, ydeficit, $Z_{e}=-v(x)$;

○ Cost C.Z $=\Sigma_{e} C_{e} \cdot Z_{e}$ is the smallest possible $\}$.

This construction yields, as in [10]:

Lemma 0. Any optimal solution (if it exists) of Load-NP-VSR provides us with an optimal loading strategy related to the route collection $\Gamma_{\text {Route }}^{*}$. 
Proof. Any loading strategy related to the tour collection $\Gamma^{*}$ may be turned into a feasible solution of Load-VSR whose cost is exactly the vehicle riding time: $\Sigma_{k}, \Sigma_{j}\left(\operatorname{DIST}\left(x(k){ }_{j}\right.\right.$, $\left.\left.x(k)_{j+1}\right) \cdot L_{j}^{*}\right)$. Conversely, any flow vector $Z$ which is a feasible solution of Load-NP-VSR can be interpreted as a loading strategy.

It comes that Non Preemptive VSR may be reformulated:

Non Preemptive VSR Reformulation: $\left\{\right.$ Compute $\Gamma_{\text {Route }}^{*}=\left\{\Gamma_{\text {Route }}(1) \ldots, \Gamma_{\text {Route }}(K)\right\}$, together with an optimal Load-NP-VSR solution $Z\left(\Gamma_{\text {Route }}^{*}\right)$, which minimize: $\left.\alpha . K+\delta . C . Z\left(\Gamma_{\text {Route }}^{*}\right)+\beta . \Sigma_{k} L-\operatorname{COST}\left(\Gamma_{k}\right)\right\}$.

\subsection{Preemptive VSR Model}

In case preemption is allowed, then we say that the VSR tour $\Gamma$ is preemptive VSR feasible if $(\mathrm{E} 1, \mathrm{E} 2, \mathrm{E} 3)$ are true. Besides, for any collection $\Gamma^{*}=(\Gamma(k), k=1 . . K \leq K$-Max $)$ of such non-preemptive feasible tours, we set, for any time value $t$, and any station $x$ :

$-\Delta(\Gamma, x, t)=\left\{(k, j), k=1 \ldots K, j=0 \ldots n(\Gamma(k))\right.$, such that $x(k)_{j}=x$ and $\left.T(k)_{j} \leq t\right\} ;$

$-H(\Gamma, x, t)=\operatorname{Sup}(0, v(x))-\Sigma_{(k, j) \in \Delta(\Gamma, x, t)} L(k)_{j}$.

Clearly, $H(\Gamma, x, t)$ denotes the number of vehicles which are really located in station $x$ at time $t$ after all loading/unloading transactions have been performed. Then we say that the collection $\Gamma=(\Gamma(k), k=1 \ldots K \leq K-$ Max) is a feasible solution for the preemptive VSR instance (X, $v, C, C A P, T$-Max, DIST, COST) if every $\Gamma(k)$ is preemptive feasible, if (E5) holds and if, for any time value $t$ and any station $x: 0 \leq H(\Gamma, x, t) \leq C(x)$.

(E6) expresses the fact that, at any time $t$, the number of vehicles currently located at $x$ is non negative and cannot exceed the capacity of the station $x$. Then we may set:

Preemptive VSR Model: $\left\{\right.$ Compute a preemptive VSR feasible tour collection $\Gamma^{*}=(\Gamma(k), k=$ $1 \ldots K)$ such that (E1, E2, E3, E5 and E6 hold) and which minimizes the following global cost:

- Global-Cost $\left(\Gamma^{*}\right)=\alpha \cdot K+\beta . \Sigma_{k} L-E-\operatorname{COST}(\Gamma(k))$

$\left.+\delta .\left(\Sigma_{k} \Sigma_{j}\left(D I S T_{x(k) j, x(k) j+1} \cdot L^{*}(k)_{j}\right)\right)\right\}$.

Remark 3. The use of preemption leads to the introduction of synchronization mechanisms. A carrier $k$ which arrives at some station $x$ may wait for another vehicle $k^{\prime}$ before leaving $x$. So L-E-COST cannot any more be replaced by L-COST in above Global-Cost. The role of the Extended Cost Hypothesis is that there is no difference, from the Global-Cost point of view, between moving from some station $x$ until some station $y$ according to a maximal speed strategy and next waiting some time $t$ at $y$, and moving from $x$ to $y$ at a reduced speed in order to arrive in $y$ with a delay $t$. Also, the vehicle riding time $\Sigma_{k} \Sigma_{j}\left(\operatorname{DIST}_{x(k) j, x(k) j+1} . L_{j}^{*}\right)$ quantity expresses the time vehicles spend into the carriers: in case some carrier $k$ arrives to some station $x$ at time 
$T$ and leaves it at time $T+t$, vehicles unloaded at time $t$ and loaded again at time $T+t$ (provided $C(x)$ is large enough) are not involves in this vehicle riding time since they are available for users between $T$ and $T+t$.

Remark 4. Taken together, above Non Preemptive and Preemptive VSR models extend [5, 7, 10, $11,14,25]$, since they unify preemption and non preemption, and mix carrier numbers, vehicle riding time and carrier riding cost into a same criterion. Still, in case of non feasibility, we do not take into account, as in $[10,11]$, the eventual deviation between the wanted balanced state and the true state of the system at the end of the process.

\subsection{A Network Flow Framework}

Let us recall that a flow vector defined on a network $G=(N, A)$, with node set $N$ and $\operatorname{arc}$ set $A$, is a rational (or integral) valued $A$-indexed vector $g$ such that, for any node $z$, the following flow conservation law holds:

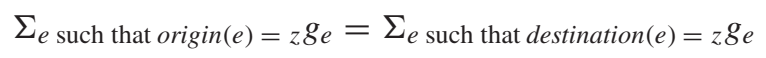

Let us now suppose that all values $D I S T_{x, y}$ are integral (it is always possible to do it). Then we derive from the VSR instance (X,v,C,CAP, T-Max, DIST, COST) a dynamic network [4] $G_{T-M a x}=\left(X_{T-M a x}, E_{T-M a x}\right)$ as follows (see Fig. 2):

- $X_{\text {T-Max }}$ is the set of all pairs $(x, t), x \in X, t=0 \ldots T$-Max, augmented with 2 nodes $s$ (source) and $p(\operatorname{sink})$;

- E-Max includes:

- Input $\operatorname{arcs}(s,(x, 0))$ and $((x, T-M a x), p)$, with null vehicle and carrier costs;

$\circ$ idle arcs $((x, t),(x, t+1))^{\text {Out }}$, with null vehicle and carrier costs;

$\circ$ carrier-idle arcs $((x, t),(x, t+1))^{I n}$ with unit vehicle costs and carrier costs equal to $\beta$.Idle-Cost if $x \neq$ Depot and 0 else;

$\circ$ active $\operatorname{arcs}\left((x, t),\left(y, t+D I S T_{x, y}\right)\right.$, with vehicle costs equal to $\delta . D I S T_{x, y}$ and carrier cost equal to $\beta \cdot C O S T_{x, y}$;

○ backward arc $(p, s)$ with null vehicle costs and carrier costs equal to $\alpha$.

Then, we may set on this network the following multi-commodity flow model:

Network-Flow-VSR Model: \{Compute non negative integral flow vectors $F$ and $f$, respectively carrier and vehicle flow vectors, such that:

○ For any idle arc $e=((x, t),(x, t+1))^{\text {Out }}, f_{e} \leq C(x)$ and $F_{e}=0$;

- For any carrier-idle arc $e=\left((x, t),(x, t+1)^{I n}, f_{e} \leq C A P . F_{e}\right.$; 


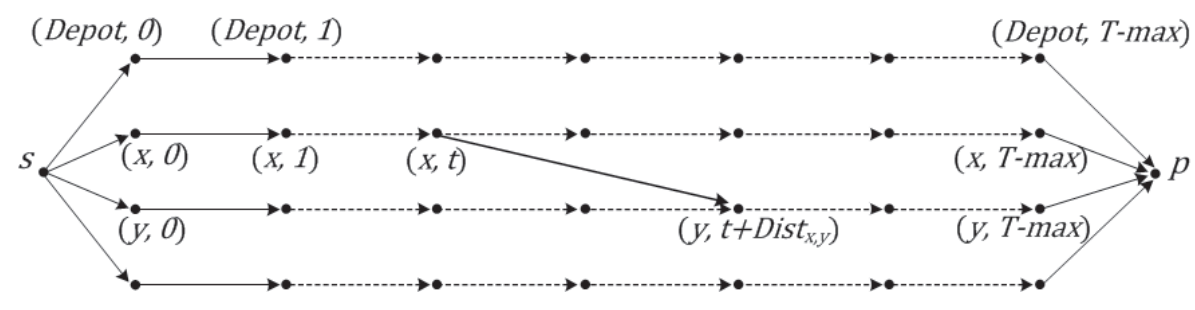

Figure 2 - Dynamic Network $G_{T-M a x}=\left(X_{T-M a x}, E_{T-M a x}\right)$.

○ For any active arc $e=\left((x, t),\left(y, t+D I S T_{x, y}\right), f_{e} \leq C A P . F_{e}\right.$;

○ For any $x \neq$ Depot, $F_{(s,(x, 0))}=F_{((x, T-M a x), p)}=0$;

$\circ$ For any node $x \neq$ Depot, $f_{(s,(x, 0))}=\operatorname{Sup}(v(x), 0)$ and $f_{((x, T-M a x), p)}=\operatorname{Sup}(-v(x), 0)$;

- $F$ and $f$ minimize $\operatorname{Cost}_{T-M a x}(F, f)=\Sigma_{e_{\in} E T-M a x} F_{e}$.Carrier-Cost C $_{e}$ $+\Sigma_{e \in E T-M a x} f_{e}$.Vehicle-Cost $\left.e_{e}\right\}$

Explanation: Flow Conservation law expresses the circulation of carriers and vehicles between the stations. Carrier-idle and idle arcs make the difference between vehicles which are waiting at some station $x$ while being located either inside some carrier or outside. (E9) says that any vehicle moving between 2 stations $x, y$ must be contained into some carrier. (E10, E11) provide us with initial and final states of both carriers and vehicles.

Theorem 1. Solving Network-Flow-VSR is equivalent to solving Preemptive VSR.

Proof. We first notice that, if a feasible preemptive VSR tour collection $\Gamma^{*}=(\Gamma(k), k=$ $1 . . K \leq K$-Max) is given, then the Extended Cost Hypothesis implies that inequalities (E1) may be supposed to be tight in case $x_{i} \neq x_{i+1}$. It comes that $\Gamma$ may be turned into a feasible NetworkFlow-VSR solution $F, f$, with same cost, by setting:

$-F_{(p, s)}=K$

- For any carrier-idle arc $e=((x, t),(x, t+1))^{I n}$ :

- $F_{e}=$ number of carriers $k$ located in $x$ between $t$ and $t+1$ according to the tours $\Gamma(k)$;

$\circ f_{e}=$ the sum of all quantities $L^{*}(k)_{j}$, taken for all carriers $k$ as above and $j$ such that $x(k)_{j}=x, x(k)_{j+1}=x, T(k)_{j} \leq t, T(k)_{j+1}>t$;

- For any idle arc $e=((x, t),(x, t+1))^{\text {Out }}: F_{e}=0$ and $f_{e}=H(\Gamma, x, t)$;

- For any active arc $e=\left((x, t),\left(y, t+D I S T_{x, y}\right), x \neq y\right.$ : 
○ $F_{e}=$ number of carriers $k$ such that $\Gamma(k)$ involves a move from $x$ to $y$ at time $t$;

$\circ f_{e}=$ sum of all $L^{*}(k)_{j}$, for all $k$ as above and $j$ such that $x(k)_{j}=x$, $x(k)_{j+1}=y, T(k)_{j}=t$;

- For any arc $e=(s,(x, 0))(e=($ Depot, T-Max $), p), F_{e}$ and $f_{e}$ are defined according to (E10) and (E11).

Conversely, if $(F, f)$ is some Network-Flow-VSR feasible solution, then we know that $F$ may be decomposed as a sum of $\{0,1\}$-valued flow vectors $F(k), k=1 \ldots K=F_{(p, s)}$. Those elementary flow vectors $F(k), k=1 \ldots K$, define in a canonical way routes $\Gamma(k)_{\text {Route }}$, together with date sequences $\Gamma(k)_{\text {Time }}$. Then, for any arc $e=\left((x, t),\left(y, t+D I S T_{x, y}\right)\right.$ and $e=$ $((x, t),(x, t+1))^{I n}$, we decompose $f_{e}$ as a sum of non negative values $f_{e}(k)$, with values no more than $C A P$. This allows us to deduce loading sequences $\Gamma(k)_{\text {Load }}=\left\{L(k)_{0}, L_{1}, \ldots, L(k)_{n(\Gamma k))}\right\}$, $k=1 \ldots K$, according to a basic $j=0, \ldots, n(\Gamma(k)$ indexed iterative process. We easily check that the resulting tour collection $\Gamma(k), k=1 \ldots K$ is preemptive VSR feasible, with a global cost Global-Cost $\left(\Gamma^{*}\right)$ exactly equal to $\operatorname{Cost}_{T-M a x}(F, f)$.

Let us now try to extend Lemma 0 to this framework. In order to do it, we consider a carrier flow vector $F$ and denote by $X(F)$ the node subset of $X_{T-M a x}$ which contains $s, p$, all nodes $(x, 0)$ and $(x, T$-Max $)$, together with all nodes $(x, t)$ which are origin or extremity of some arc $e=\left((x, t),\left(y, t+D I S T_{x, y}\right), x \neq y\right.$, such that $F_{e} \neq 0$. We provide $X(F)$ with an arc set $E(F)$ which contains $\operatorname{arcs}(s,(x, 0)),(x, T-M a x), p), x \in X$, as well as:

- related active $\operatorname{arcs} e=\left((x, t),\left(y, t+D I S T_{x, y}\right), x \neq y\right.$;

- extended idle arcs $\left((x, t),\left(x, t^{\prime}\right)\right)^{I n}$ and $\left((x, t),\left(x, t^{\prime}\right)\right)^{\text {Out }}$, with $t, t^{\prime}$ such that no $\left(x, t^{\prime \prime}\right)$ exists in $X(F)$ such that $t<t^{\prime}<t^{\prime \prime}$; those arcs are provided with vehicle-cost values respectively equal to $\left(t^{\prime}-t\right)$ and 0 ;

Since values $F_{e}$ defined on idle $\operatorname{arcs} e=((x, t),(x, t+1))^{I n}$ can be turned in a natural way into values $F_{e}$ defined on extended idle arcs $\left((x, t),\left(x, t^{\prime}\right)\right)^{I n}, F$ may be viewed as a flow vector on the network $(X(F), E(F))$. Then we set:

Load-P-VSR: $\{$ Compute, on the network $(X(F), E(F))$ a non negative flow vector $f$, such that:

- For any active arc $e=\left((x, t),\left(y, t+\operatorname{DIST}_{(x, y)}, x \neq y\right.\right.$ and any extended-idle arc

$$
e=\left((x, t),\left(x, t^{\prime}\right)\right)^{I n} \text { we have } f_{e} \leq C A P . F_{e} ;
$$

- For any station $x, f_{(s,(x, 0))}=\operatorname{Sup}(v(x), 0)$ and $f_{((x, T-M a x), p)}=\operatorname{Sup}(-v(x), 0)$;

- $f$ minimizes the linear cost $\Gamma_{e \in E} f_{e}$.Vehicle-Cost $\left.t_{e}\right\}$ 
This allows us to state the following extension of Lemma 0 (proof left to the reader):

Lemma 1. F being given, solving Load-P-VSR provides us with an optimal loading strategy.

One may now ask about casting Non Preemptive VSR into this Network Flow framework. This leads us to set:

NP-Network-Flow-VSR Model: \{Compute $K$ and two non negative integral multi-commodity flow integral vectors $F=(F(k), k=1 \ldots K)$ and $f=(f(k), k=1 \ldots K)$, respectively carrier and vehicle multi-commodity flow vectors, such that:

○ For any $k, F(k)$ is $\{0,1\}$-valued;

○ For any idle arc $e=((x, t),(x, t+1))^{I n}$, $x \neq$ Depot, $\Sigma_{k} f(k)_{e}=\Sigma_{k} F(k)_{e}=0 ;$

- For any idle arc $e=((x, t),(x, t+1))^{\text {Out }}$, $x \neq$ Depot, $\Sigma_{k} F(k)_{e}=0$;

○ For any idle arc $e=((\text { Depot }, t),(\text { Depot }, t+1))^{\text {Out }}, \Sigma_{k} f(k)_{e}=0$;

○ For any active arc $e=\left((x, t),\left(y, t+D I S T_{x, y}\right), f(k)_{e} \leq C A P \cdot F(k)_{e}\right.$;

○ For any $x \neq$ Depot, $\Sigma_{k} F(k)_{(s,(x, 0))}=\Sigma_{k} F(k)_{((x, T-M a x), p)}=0$;

○ For any $x \neq$ Depot, $\Sigma_{k} f(k)_{(s,(x, 0))}=\operatorname{Sup}(v(x), 0)$ and $\Sigma_{k} f(k)_{((x, T-M a x), p)}=\operatorname{Sup}(-v(x), 0)$;

- For any $k$ and any excess (deficit, neutral) station $x$, values

$$
f(k)_{e}, e=\left((x, t),(x, t+1)^{I n},\right.
$$

are decreasing (increasing, stationary) when t increases;

○ $F$ and $f$ minimize $\Sigma_{e \in E T-M a x} F_{e}^{*}$.Carrier-Cost $e_{e}+\Sigma_{e \in E T-M a x} f_{e}^{*}$.Vehicle-Cost $\left.t_{e}\right\}$

Theorem 2. Solving NP-Network-Flow-VSR is equivalent to solving Non Preemptive VSR.

Proof. It is pure routine to check that any Non Preemptive VSR feasible solution $\Gamma^{*}$ gives rise to a feasible solution $(F, f)$ of NP-Network-Flow-VSR with the same cost value. Conversely, monotony constraint (E17) forbids any carrier $k$ from unloading (loading) at some excess or neutral (deficit or neutral) station $x$, enabling us to turn flow vector $f(k)$ into a loading strategy for the tour $\Gamma(k)$ induced by $\{0,1\}$-valued flow vector $F(k)$.

Remark 5. Those reformulations make clearly appear that Preemption is a relaxation of Non Preemption. 


\section{VSR LOWER BOUNDS}

We propose here 2 classes of easy to compute vehicle driven lower bounds for the VSR Problem: the first one relies on Min-Cost Assignment models which separately bound the active carrier number, the carrier riding cost and the vehicle riding time. The second one directly derives from the previous Network-Flow-VSR model.

\subsection{Min-Cost Assignment Based Lower Bounds}

Let us consider the following ILP models:

VMCA Vehicle-Min-Cost-Assign: $\left\{\right.$ Compute integral vector $Q=\left(Q_{x, y}, x\right.$ excess, $y$ deficit $) \geq$ 0, such that:

○ For any excess station $x, \Sigma_{y}$ deficit station $Q_{x, y}=v(x)$

○ For any deficit station $y, \Sigma_{x}$ excess station $Q_{x, y}=-v(y)$

○ Minimize $\left.\Sigma_{x, y} \operatorname{DIST}_{x, y} \cdot Q_{x, y}\right\}$

$L B-V M C A$ denotes the related optimal value, which may be computed while relaxing the integrality constraint on the vector $Q$. In any case (preemption or not), $L B$-VMCA provides us with a lower bound of the vehicle riding time: $\Gamma_{k} \Sigma_{j}\left(D I S T_{x(k) j, x(k) j+1} \cdot L_{j}^{*}\right)$.

CMCA Carrier-Min-Cost-Assign: \{Compute integral vector $R=\left(R_{x, y}, x, y\right.$ stations $) \geq 0$, such that:

○ For any neutral station $x \neq$ Depot, $\Sigma_{y} R_{x, y}=0=\Sigma_{y} R_{y, x}$

○ For any excess station $x, C A P . \Sigma_{y} R_{x, y}=C A P . \Sigma_{y} R_{y, x} \geq v(x)$

- For any deficit station $y, C A P . \Sigma_{x} R_{x, y}=C A P . \Sigma_{x} R_{y, x} \geq-v(y)$

○ $\Sigma_{y} R_{\text {Depot }, y}=\Sigma_{y} R_{y, \text { Depot }} \geq 1$

○ Minimize $\left.\Sigma_{x, y} \operatorname{COST}_{x, y} \cdot R_{x, y}\right\}$

$L B$ - $C M C A$ denotes the related optimal value, which may be computed in polynomial time through a simple Min Cost Flow algorithm. $L B-C M C A$ is a lower bound for the carrier riding cost $\Sigma_{k} L-E-C O S T(\Gamma(k))$. If LB-Time-CMCA is the value of the CMCA model obtained by replacing the COST matrix by the DIST matrix, then LB-Time-CMCA/T-Max is a lower bound for the carrier number $K$.

UCMCA Unit-Carrier-Min-Cost-Assign: $\left\{\right.$ Compute rational vector $R=\left(R_{x, y}, x\right.$ stations $) \geq 0$, such that: 
○ For any neutral station $x \neq$ Depot, $\Sigma_{y} R_{x, y}=0=\Sigma_{y} R_{y, x}$

○ For any $x, y$, both deficit or both excess, $R_{x, y}=0$

- For any y deficit, $R_{\text {Depot }, y}=0$ and for any $x$ excess, $R_{x, \text { Depot }}=0$

○ For any excess station $x, \Sigma_{y}$ deficit or Depot $R_{y, x}=\Sigma_{y}$ deficit $R_{x, y}=v(x)$

○ For any deficit station $y, \Sigma_{x \text { excess or Depot }} R_{y, x}=\Sigma_{x \text { excess }} R_{x, y}=-v(y)$

○ $\Sigma_{y \text { excess }} R_{\text {Depot }, y}=\Sigma_{y} R_{y}$ deficit,Depot $=1$

○ For any subset $A \subseteq X-\{$ Depot $\}, A \neq N i l, \Sigma_{x \in A y, y \notin A} R_{x, y} \geq 1$ (No-Subtour Constraint)

○ Minimize $\left.\Sigma_{x, y} \operatorname{COST}_{x, y} \cdot R_{x, y}\right\}$

$L B$-UCMCA denotes the related optimal value. We see that $L B-U C C A$ is a lower bound for the $L-C O S T$ value of any tour $\gamma$ which starts and ends into Depot, while alternatively moving from excess nodes to deficit nodes and carrying unit loads. If LB-Time-UCMCA is the optimal value of the UCMCA model obtained by replacing the COST matrix by the DIST matrix, then LB-Time$U C M C A$ is a lower bound for the carrier riding time L-DIST induced by $\gamma$. UCMCA involves significantly less variables than the $C M C A$ model.

We deduce:

Theorem 3. A VSR (Preemptive or Not) lower bound is given by LB-MCA $=\alpha$ LB-Time$C M C A / T-M a x+\beta . L B-C M C A+\delta . L B-V M C A$.

Proof. It is contained into the comments which come together with the definition of the above models.

Theorem 4. A Non Preemptive VSR lower bound is given by LB-UMCA $=\alpha$ LB-Time-UCMCA/ $(C A P . T-M a x)+\beta . L B-U C M C A / C A P+\delta . L B-V M C A$.

Proof. Any tour $\gamma$ which satisfies (E1, E2, E3, E4) may be split into CAP tours $\gamma_{1}, \ldots, \gamma_{C A P}$, all with same lengths, which globally perform the relocation process when related $C A P=1$. It comes from the fact that any solution $Z$ of the LOAD-NP-VSR model related to $\gamma$ may be decomposed into a sum $Z_{1}+\cdots+Z_{C A P}$ of $\{0,1\}$-valued flow vectors. So, if Carrier-Ride-Time 1 and Carrier-Ride-Cost ${ }_{1}$ respectively denote the smallest possible values for the carrier riding time and the carrier riding cost related to the case when $C A P=1$ and $T$-Max $=+\infty$, we see that: the carrier riding time (carrier riding cost) of any solution $\Gamma$ of Non Preemptive VSR is at least equal to Carrier-Ride-Time ${ }_{1} / C A P$ (Carrier-Ride-Cost $\left.1 / C A P\right)$. We deduce that $\alpha$. $\lceil$ CarrierRide-Time $_{1} /$ CAP.T-Max $\rceil+\beta$ Carrier-Ride-Cost $1 / C A P+\delta . L B-V M C A$ is a Non Preemptive VSR lower bound. But Carrier-Ride-Time 1 corresponds to a kind of TSP carrier tour starting and ending into Depot, according to which the carrier alternatively moves from excess to deficit nodes. Clearly LB-Time-UCMCA provides us with a lower bound for the DIST-length of such a tour. The same reasoning holds with Carrier-Cost-Time 1 . So we conclude. 


\subsection{Projected Flow Lower Bound}

We derive from the dynamic network $G_{T-M a x}=\left(X_{T-M a x}, E_{T-M a x}\right)$ of Section 2.4 a projected network $G_{P r o j}=\left(X_{P r o j}, E_{P r o j}\right)$ as follows (see Fig. 3):

$\circ X_{\text {Proj }}=X \cup\{s, p\}$ where nodes $s$ and $p$ are additional nodes source and sink;

- The restriction of $G_{P r o j}$ to $X$ is a complete network: any $\operatorname{arc} e=(x, y)$ is provided with a carrier cost $C C_{e}=\beta \cdot C O S T_{x, y}+(\alpha / T$-Max $) . D I S T_{x, y}$ and with a vehicle cost $C V_{e}=$ $\delta . D I S T_{x, y}$.

- There is an $\operatorname{arc}(s, x)$ from $s$ to any excess station $x$, with null carrier and vehicle costs;

- There is an arc $(y, p)$ from any deficit station $y$ to $p$, with null carrier and vehicle costs;

- There is a backward arc $(p, s)$, with null carrier and vehicle costs.

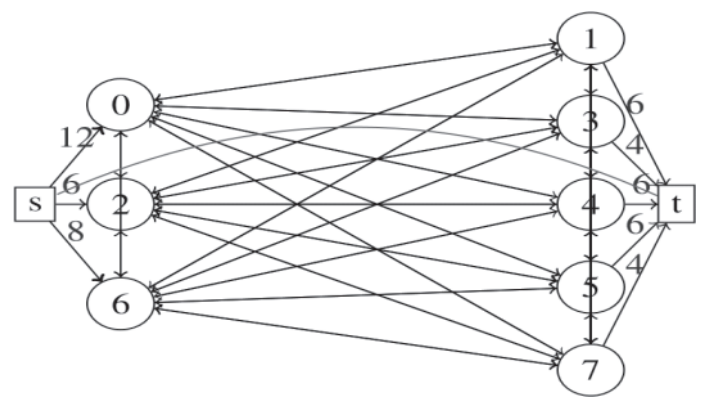

Figure 3 - A network $G_{\text {Proj }}$ derived from 3 excess stations and 5 deficit stations.

Then we set:

Projected-VSR-Flow Model: $\left\{\right.$ Compute on the network $G_{P r o j}$ two integral flow vectors $H$ and $h$ such that:

○ For any $\operatorname{arc} e=\left((x, y), x, y \neq s, p, h_{e} \leq C A P . H_{e}\right.$

- For any excess (or neutral) station $x, h_{(s, x)}=v(x)$ and

for any deficit station $y, h_{(y p)}=-v(x)$

$\circ \Sigma_{y} H_{\text {Depot }, y}=\Sigma_{y} H_{y, \text { Depot }} \geq 1$

○ Minimize $\left.\Sigma_{e} C C_{e} . H_{e}+\Sigma_{e} C V_{e} \cdot h_{e} \cdot\right\}$

We denote by $L B$-Proj-Flow the related optimal value of this program. Then we state:

Theorem 5. LB-Proj-Flow is a (Preemptive or Not) VSR lower bound, such that LB-Proj-Flow $\geq L B-M C A$. 
Proof. Any feasible solution $F, f$ of Network-Flow-VSR can be turned into a feasible solution $H, h$ of Projected-VSR-Flow. The cost $\operatorname{Cost}_{\text {T-Max }}(F, f)=\Sigma_{e \in E T-M a x} F_{e}$.Carrier-Cost Cl $_{e}+$ $\Sigma_{e \in E T-M a x} f_{e}$.Vehicle-Cost $t_{e}$ may be decomposed into:

$\operatorname{Cost}_{T-M a x}(F, f)=\alpha \cdot F_{(p, s)}+\Sigma_{e \in E T-M a x, e \neq(p, s)} F_{e}$. Carrier-Cost $t_{e}+\Sigma_{e \in E T-M a x} f_{e}$. Vehicle-Cost ${ }_{e}$.

Through projection, the two last terms of this sum give rise to the quantity $\Sigma_{\text {arcs } e} \beta . C O S T_{e} . H_{e}+$ $\Sigma_{\text {arcs } e} C V_{e} . h_{e}$. The first component corresponds to $\alpha . K$, where $K=F_{(p, s)}$ is the carrier number. But we know that this carrier number is at least equal to $\left(\Sigma_{e=(x, y) \in E-P r o j} H_{x, y}\right.$.DIST $\left.x, y\right) / T$-Max. We deduce the first part of our statement.

As for the second part, we get it by noticing that any solution $H, h$ of the Projected-VSR-Flow program give rise in a natural way to a feasible solution $R$ of the $C M C A$ program and a feasible solution $Q$ of the VMCA program, and by keeping on with the above decomposition of the quantity $\Sigma_{\text {arcs } e} C C_{e} \cdot H_{e}+\Sigma_{\text {arcse }} C V_{e} \cdot h_{e}$.

Remark 6. The Projected-VSR-Flow model does not solve our VSR problem, even according to its preemptive version. For instance one may consider a station set $X=\{$ Depot, $A, B, C\}$, a carrier flow $H$ related to the route (Depot, $A, B, C, A$, Depot) followed by 1 carrier with capacity 1 , and a vehicle flow $h$ which routes 1 flow unit from excess station $C$ to deficit station $B$. Then the carrier cannot deliver its load in $B$ before picking it up in $C$.

Remark 7. $L B$-Proj-Flow value provides us with a better lower bound than the $L B$-MCA lower bound of Theorem 3. Still, Projected-VSR-Flow is a complex NP-Hard model, whose rational relaxation yields a poor lower bound as soon as CAP is large. The Lagrangean relaxation of the coupling constraint (E18) yields a Lagrangean value $\operatorname{Sup}_{\lambda \in \Lambda}\left(\operatorname{Inf}_{h}(C V+\lambda) \cdot h\right)+\operatorname{Inf}_{H}(C C-$ $\lambda) . H$ ) where:

- Vector flow $h$ is subject to (E19) and vector flow $H$ is subject to (E20);

$\circ \Lambda=\left\{\lambda\right.$ such that the restriction of the graph $G_{P r o j}$ to $X$ does not contain any negative $(C C-\lambda)$-circuit\}.

But, because of the total unimodularity of flow constraint matrices, this value is the same as the value obtained by performing Lagrangean relaxation of (E18) on the rational relaxation of Projected-VSR-Flow. That means that the above Lagrangean value does not improve the standard relaxation of the integrality constraint.

\section{A VEHICLE MIN-COST ASSIGNMENT BASED HEURISTIC FOR NON PREEMPTIVE VSR}

We focus here on Non Preemptive VSR, and derive from the LB-MCA lower bound a decomposition of this problem into a Master Vehicle-Min-Cost Assignment problem and a Slave Pickup\&Delivery $(P D P)$ Problem. 


\subsection{MCA/PDP Decomposition}

Let us recall that a Pick-up\&Delivery instance (see $[2,12,17])$ is defined by:

○ a set $J$ of requests $j=(o(j), d(j), \lambda(j))$, where $o(j), d(j)$ and $\lambda(j)$ are respectively the origin, the destination and the load of $j ; N$ denotes the set of all nodes $o(j), d(j), j \in J$, augmented with a Depot node and considered as pairwise distinct; these requests have to be served by trucks, initially located in Depot and all with capacity $\mathrm{CH}$;

- 2 distance matrices $D$ and $C S$, indexed on the set $N . N$ and a threshold D-Max;

- Scaling coefficients $A, B, C$.

A collection $\rho$ of truck routes $\rho(m), m=1 \ldots M$ defined on the set $N$ is a feasible PDP solution if:

o every request $j$ is serviced by some truck $m: m$ first loads $\lambda(j)$ at $o(j)$ and unloads it into $d(j)$

○ the load of a truck never exceeds capacity $\mathrm{CH}$;

○ the $D$-length of $\rho(m)$ of any truck route $\lambda(m), m=1 . . M$, never exceeds $D$-Max.

It is an optimal $P D P$ solution if it is feasible and minimizes a quantity:

$$
\operatorname{PDP}-\operatorname{COST}(\rho)=A \cdot M+B \cdot \Sigma_{m} C S-\operatorname{Length}(\rho(m))+C \cdot \Sigma_{j} \lambda(j) \cdot \operatorname{D-Ride}(j),
$$

where $D$-Ride $(j)$ is the $D$-length which is run by load $\lambda(j)$ inside a truck. A Load-Split PDP instance is defined the same way, but every loads $\lambda(j)$ may be split into a sum $\lambda(j)=\lambda(j)_{1}+$ $\cdots+\lambda(j)_{Q(j)}$, of several sub-loads, which are separately handled.

Though Load-Split PDP is NP-Hard, it may be in practice efficiently handled through a GRASPVNS (Greedy Randomized Adaptative Search + Variable Neighborhood Search) process based upon Insert/Remove operators:

- Insert operator: Inserting request $j=(o(j), d(j), \lambda(j))$ into some truck route $\rho(m)$ means:

- computing 2 insertion nodes $x$ and $y$ in $\rho(m)$, and some sub-load $\lambda \leq \lambda(j)$;

$\circ$ inserting $o(j)(d(j))$ between $x(y)$ and its successor in $\rho(m)$;

$\circ$ adding $\lambda$ to the current load of $\rho(m)$ between $x$ and $y$, and updating $\lambda(j)$;

- Remove operator: Delete $o(j)$ and $d(j)$ from $\rho(m)$ and update the load of $m$ and the $\lambda(j)$ value accordingly. 
Then related GRASP-VNS scheme comes as follows:

\section{PDP GRASP-VNS Algorithm}

\section{Randomized Initialization:}

While all requests have not been inserted do

Randomly pick up some non inserted request $j$;

Compute (in a heuristic way) truck parameter $m$, together with insertion parameters $x, y \in \rho(m)$, and $\lambda \leq \lambda(j)$ in such a way that related insertion is feasible and such that (bi-criteria choice):

o the induced increase of $\operatorname{PDP}-\operatorname{COST}(\rho)$ is the smallest possible;

$\circ \lambda$ is the largest possible;

Local Search Loop:

Not Stop;

While Not Stop do

Identify a set $J_{0} \subseteq J$ of poorly inserted requests;

Remove $J_{0}$ from $J$ and reinsert it according to the same process as in the initialization;

Update the current best solution $\rho^{*}=(\rho(m), m=1 . . M)$; Update Stop.

Let us now come back to our Non Preemptive VSR instance, and suppose that, for some instance (X, v, C, CAP, T-Max, DIST, COST), we know, for every pair $(x, y), x$ excess, y deficit station, which quantity $Q_{x, y}$ has to move from $x$ to $y$. Then, we only need to solve the Load-Split PDP instance defined by:

○ Requests $j$ are all 3-uples $\left(o(j)=x, d(j)=y, \lambda(j)=Q_{x, y}\right)$, taken for all pairs $x, y$ such that $Q_{x, y} \neq 0$;

○ $D-M a x=T-M a x ; D=D I S T ; C S=C O S T ; C H=C A P ; A=\alpha, B=\beta, C=\delta$.

One may conjecture that it is possible to impose assignment vector $Q$ to be an optimal solution, for some cost vector $U=\left(U_{x, y}, x\right.$ Excess, y Deficit) $\geq 0$, of the following $\operatorname{VMCA}(U)$ (Vehicle Min-Cost Assignment) model:

$\boldsymbol{V M C A}(\boldsymbol{U}):\left\{\right.$ Compute integral vector $Q=\left(Q_{x, y}, x\right.$ excess, $y$ deficit stations $) \geq 0$, such that:

○ For any excess station $x, \Sigma_{y}$ deficit station $Q_{x, y}=v(x)$; For any deficit station $y$, $\Sigma_{x \text { excess station }} Q_{x, y}=-v(y)$;

○ Minimize $\left.\Sigma_{x, y} U_{x, y} \cdot Q_{x, y}\right\}$ 
Though we cannot prove this conjecture, it leads us to the following reformulation of Non Preemptive VSR:

Non Preemptive VSR VMCA Reformulation: \{Compute cost vector $U=\left(U_{x, y}, x\right.$ Excess, $y$ Deficit $) \geq 0$, such that the optimal value of the related Load-Split PDP instance be the smallest possible\}.

We may handle this reformulation through the following algorithmic scheme:

VSR-MCA Algorithm ( N : Loop Number)

Initialize cost vector $U=\left(U_{x, y}, x\right.$ Excess, y Deficit $) \geq 0$;

For $j=1 \ldots N$ do $(*$ Local Search loop*)

Derive a PDP Assignment vector $Q$ through optimal resolution of $\operatorname{VMCA}(U)$;

Solve (in a heuristic way) the related Load-Split PDP instance;

Update cost vector $U$;

Apply to the resulting route collection $\Gamma_{\text {Route }}^{*}=\left\{\Gamma_{\text {Route }}(1), \ldots, \Gamma_{\text {Route }}(K)\right\}$ the Load-NP-VSR model, clean the routes $\Gamma_{\text {Route }}(k)$ from its useless stations;

Keep the best result ever obtained.

Two critical points have to be specified inside this algorithmic description:

1) "Initialize cost vector $\boldsymbol{U}$ " instruction: LB-MCA lower bound of Section 3 suggests us to apply what we call the Shortest Distance/Cost Strategy, and set, for any $x, y, x$ Excess, $y$ Deficit, $U_{x, y}=D I S T_{x, y}+\lambda .\left(\operatorname{COS} T_{x, y} .+\operatorname{COST}_{y, x}\right)$ where $\lambda$ is some non negative coefficient; as a matter of fact, doing this leads us to extend the above VSR-MCA algorithm into a GRASP algorithmic scheme, by performing initialization of the cost vector $U$ in a random way:

GRASP-VSR-MCA Algorithm (N: Loop Number, R: Replication Number)

For $i=1 \ldots R$ do

Randomly generate $\lambda \geq 0$;

For any $x, y, x$ Excess, $y$ Deficit, $U_{x, y} \leftarrow D I S T_{x, y}+\lambda .\left(\operatorname{COST}_{x, y} .+\operatorname{COST}_{y, x}\right)$;

For $j=1 \ldots N$ do ... (*Local Search loop of VSR-MCA*);

Keep the best result ever obtained.

2) "Update cost vector U" instruction: Let us denote by $U^{0}$ the initial cost vector and let us consider that we are provided with a current cost vector $U$. We derive from $U$ a request 
vector $Q$, a request set $\operatorname{Req}(U)=\left\{r=\left(x, y, Q_{x, y}\right)\right.$ such that $\left.Q_{x, y} \neq 0\right\}$ and a Non Preemptive VSR solution $\Gamma^{*}$, whose global cost Global-Cost $\left(\Gamma^{*}\right)$ may be distributed among requests $\left(x, y, Q_{x, y}\right)$ in a natural way:

- The carrier cost $\alpha+\beta . L-\operatorname{COST}(\Gamma(k))$ related to a given carrier $k$ is shared between the requests which are served by this carrier, proportionally to the value L-COST $\left(\Gamma(k)_{x, y}\right) \cdot Q_{x, y}$, where $\Gamma(k)_{x, y}$ is the sub-route which is induced by the restriction $\Gamma(k)_{x, y}$ of $\Gamma(k)$ between $x$ and $y$ (in case $Q_{x, y}$ is split into sub-loads, we deal separately with those sub-loads);

- Every request $r=\left(x, y, Q_{x, y}\right)$ is assigned its part $L$-DIST $\left(\Gamma(k)_{x, y}\right) \cdot Q_{x, y}$ of the vehicle riding time.

It comes that Global-Cost $(\Gamma *)$ may be written Global-Cost $\left(\Gamma^{*}\right)=\Sigma_{r \in \operatorname{Req}(U)}$ Partial-Cost $\left(r, \Gamma^{*}\right)$, where Partial-Cost $\left(r, \Gamma^{*}\right)$ is the part of Global-Cost $\left(\Gamma^{*}\right)$ which is charged this way to request $r$. Then, for every request $r=\left(x, y, Q_{x, y} \neq 0\right)$ we set $V_{x, y}=\operatorname{Partial-\operatorname {Cost}}\left(r, \Gamma^{*}\right) / Q_{x, y}$ and update $U$ as follows:

- If $Q_{x, y} \neq 0, U_{x, y}$ is replaced by $\left(U_{x, y}+V_{x, y}\right) / 2$ else $U_{x, y}$ is unmodified;

- When $U=U^{0}, U$ values may be very different from $V$ values. So we compute the mean value $\tau$ of the ratio $V_{x, y} / U_{x, y}, x, y$ such that $Q_{x, y} \neq 0$, and replace every value $U_{x, y}^{0}$ by $=\tau \cdot U_{x, y}^{0}$.

\subsection{An Approximation Result}

A natural question comes about the quality of the Shortest Cost/Distance strategy. Since, in most cases, the COST and DIST matrices are strongly correlated, we consider here the case when those matrices are the same, and when Global-Cost only involves the carrier riding cost. In such a case, we may state:

Theorem 6 (Shortest Cost/Distance Strategy). If COST $=$ DIST, if $\alpha=\delta=0$ (focus on carrier riding cost minimization) and if T-Max $=+\infty$, then the Shortest Cost/Distance strategy induces an approximation ratio of $(1+C A P)$. This is the best possible ratio.

Proof. We first notice that we may, since T-Max $=+\infty$, deal with only one carrier. Let us first prove the first part of the result, that means that there is no approximation ratio better than $(1+C A P)$. In order to do so, we build the following Non Preemptive VSR instance:

$-K=1$;

$-X=\{$ Depot $\} \cup\left\{o_{n, c}, d_{n, c}, n=0 . . N-1, c=1 . . C A P\right\}$ where $N$ is a large number; function $v$ is equal to 1 for $o_{n, c}$ (excess) stations and to -1 for $d_{n, c}$ (deficit) stations; 
- DIST $=$ COST represents the shortest path distance induced on the set $X$ by the following arc set $E=E_{1} \cup E_{2} \cup E_{3} \cup E_{4}$ :

○ $E_{1}=\left\{\left(\right.\right.$ Depot,$\left.o_{0,1}\right),\left(d_{N-1,1}\right.$, Depot $\left.)\right\}$, both arcs with length equal to $1 / 2$;

$\circ E_{2}=\left\{\left(o_{n, c}, o_{n, c+1}\right),\left(d_{n, c+1}, d_{n, c}\right), n=0 . . N-1, c=1 . . C A P-1\right\}$, all arcs with small length $\varepsilon$;

$\circ E_{3}=\left\{\left(o_{n, C A P}, d_{n, C A P}\right), n=0 . . N-1\right\} \cup\left\{\left(d_{n, 1}, o_{n+1,1}\right), n=0 . . N-2\right\}$, all arcs with length 1 ;

$\circ E_{4}=\left\{\left\{\left(o_{n, c}, d_{n-1, c}\right)\right\}, n=0 . . N-1, c=1 . . C A P\right\}$ addition being performed modulo $N$, all arcs with length $1-\alpha$, where $\alpha$ is a small number.

One easily checks that an optimal tour for the carrier is the tour $\left\{\right.$ Depot, $o_{0,1}, \ldots, o_{0, C A P}$, $d_{0, C A P}, \ldots, d_{0,1}, o_{1,1, \ldots}, o_{1, C A P}, \ldots$, Depot $\}$, with length $L-D I S T=2 n+2 n .(C A P-1) \varepsilon$. For every $n=0, \ldots, N-1$, this tour makes the carrier load all the excess vehicles located in excess stations $o_{n, c}, c=1 \ldots C A P$, and next bring them to deficit stations $d_{n, c}, c=C A P \ldots 1$, before moving to node $o_{n+1,1}$. On another side, the vector $Q$ deriving from the Shortest Cost/Distance strategy is provided by $E_{4}$. One checks that a related optimal PDP meets every request related to an $\operatorname{arc}\left(o_{n, c}, d_{n-1, c}\right)$ through a direct move $\left(o_{n, c}, d_{n-1, c}\right)$ (proof left to the reader: if it were not the case, then one could remove related arcs of $E_{4}$ ). So, as soon as the carrier has been loading in station $o_{n, c}$, it moves to station $d_{n-1, c}$ and delivers its load. A consequence is that at any time during the process, the current loads of the carrier does not exceeds 1 and that the optimal PDP solu-

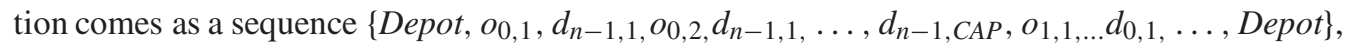
with length $L-D I S T=C A P . n(1-\alpha)+n . C A P .(1+(C A P-1) \cdot \varepsilon+2 n+n .(C A P-1) \varepsilon$. We conclude.

In order to prove the first part of the result, that means that $(1+C A P)$ provides us with an approximation ratio, we first notice that splitting any station $x$ into $v(x)$ copies, all with $v$ value equal to 1 or -1 and to distance 0 to each other does not modify the problem. Then we consider some feasible Preemptive VSR tour $\gamma=\left\{\right.$ Depot, $x_{0}, x_{1}, \ldots, x_{n(\gamma)}=$ Depot $\}$. Clearly, we may suppose that no station is involved more than once in $\gamma$. Then we may state:

Lemma 2. There cannot exist any sequence (discrete circular interval) $J=\left\{x_{i}, x_{i+1} \ldots, x_{i+t}\right\}$, addition being taken modulo $n(\gamma)$, such that $\Sigma_{x \in J} v(x) \leq C A P-1$.

Proof. If such a sequence exists then the load of the carrier just before reaching $x_{i}$ is at least equal to $C A P+1$.

Lemma 3. There exists some one-to-one involutive correspondence $u=u_{\gamma}$ from $X$ into itself such that:

- If $x$ is an excess station then $u_{\gamma}(x)$ is a deficit station and conversely;

- If one runs along $\gamma$ from some deficit station $x$, then it visits no more that CAP-1 stations other than (eventually) Depot, $x$ and $u_{\gamma}(x)$ before reaching $u_{\gamma}(x)$. We denote by $\gamma(x, u)$ the related sub-path of $\gamma$. 
By the same way there exists a one-to-one involutive correspondence $w=w_{\gamma}$ from $X$ into itself such that:

- If $x$ is an excess station then $w_{\gamma}(x)$ is a deficit station and conversely;

- If one runs along $\gamma$ from some excess station $x$, then it visits no more that CAP-1 stations other than (eventually) Depot, $x$ and $w_{\gamma}(x)$ before reaching $w_{\gamma}(x)$. We denote by $\gamma(x, w)$ the related sub-path of $\gamma$.

Proof. For any node $x=x_{i}$ of $\gamma$, we set $J_{x}=\left\{x_{i}, x_{i+1}, \ldots, x_{i+C A P}\right\}$, addition being taken modulo $n(\gamma)$. Then, we build a bipartite graph $(U, V, E)$ by setting:

- $U=\{$ deficit stations of $\gamma\} ; V=\{$ excess stations of $\gamma\}$;

- $E=\left\{\left(x_{i}, x_{j}\right)\right.$ such that one visits no more than $C A P-1$ non trivial stations when running from $x_{i}$ to $x_{j}$ along $\left.\gamma\right\}$.

The first part of Lemma 3 (existence of $u=w_{\gamma}$ ) means that this bipartite graph admits a perfect matching. If it is not true, then Koenig-Hall Theorem tells us that there exists $U^{*} \subseteq U$ such that $\operatorname{Card}\left(\left\{v \in V\right.\right.$ which are the extremity of an edge $\left.\left.(u, v), u \in U^{*}\right\}\right) \leq \operatorname{Card}\left(U^{*}\right)-1$. One may choose $U^{*}$ in such a way that the intersection graph defined by the discrete circular intervals $J_{x}, x \in U^{*}$ is connected. But then we see that the discrete interval $J=\cup_{x \in U^{*}} J_{x}$ is such that $\Sigma_{x \in J} v(x) \leftarrow C A P$, and thus that it contradicts former Lemma 2. We proceed the same way in order to get the existence of $w=w_{\gamma}$.

Lemma 4. A same transition $x_{i} \rightarrow x_{i+1}(i+1$ being computed modulo $n)$ of $\gamma=\left\{\right.$ Depot, $x_{0}$, $x_{1}, \ldots, x_{n}=$ Depot $\}$, cannot appear more than CAP times in the path collection $\{\gamma(x, u)$, $\gamma(x, w), j=0, \ldots, n-1\}$ of Lemma 3 .

Proof. If the transition $x_{i} \rightarrow x_{i+1}$ is involved into $\gamma(x, u)$ then $x$ is a deficit station and is one of the CAP stations which are located before in $\gamma$. If it is involved into $\gamma(x, w)$ then $x$ is an excess station and is one of the CAP stations which are located before in $\gamma$. We conclude.

We may now finish with the proof of Theorem 6. Let us suppose that tour $\gamma$ is an optimal solution of Non Preemptive VSR and that we are provided with a min-cost assignment $Q$, which, with any excess station $x$, associates some deficit station $z_{Q}(x)$ in a one-to-one way and which is such that $\Sigma_{x \text { excess }} D I S T_{x, Q(x)}$ is the smallest possible. Then for any excess station $x$, we may derive a circuit $\gamma(x)$ as follows: Start from $x$, then go to the deficit node $z_{Q}(x)$, next go to $u_{\gamma}(x)$ of Lemma 3 while following $\gamma$ and keep on this way. Two circuits $\gamma(x)$ and $\gamma(y)$ are either identical or disjoint, and induce a partition of $X-\{$ Depot $\}$ into a collection $\left\{\gamma_{1}, \ldots, \gamma_{P}\right\}$ of circuits, with related representative stations $x^{p}$ labeled in such a way that they come according to this order in the tour $\gamma$. 
Then we derive a new Non Preemptive VSR solution $\gamma^{*}$ as follows: Start from Depot, go to $\gamma_{1}$ representative station $x^{1}$ along $\gamma$, run along $\gamma_{1}$, next go to $x^{2}$ along $\gamma$ and so on until going back to Depot after running $\gamma_{P}$.

The length $\operatorname{L-DIST}\left(\gamma^{*}\right)$ is equal to $\operatorname{L-DIST}(\gamma)+\Sigma_{p} \operatorname{L-DIST}\left(\gamma_{p}\right)$. But we also have $\Sigma_{p} L$ $\operatorname{DIST}\left(\Sigma_{p}\right)=\Sigma_{x \text { deficit }} \operatorname{L-DIST}(\gamma(x, u))+\Sigma_{x \text { excess }} \operatorname{DIST}_{x, z Q(x)} \leq \Sigma_{x \text { deficit }} \operatorname{L-DIST}(\gamma(x, u))+$ $\Sigma_{x \text { excess }} \operatorname{L-DIST}(\gamma(x, w))$. Because of Lemma 4, a same transition $x_{i} \rightarrow x_{i+1}$ of $\gamma=\left\{x_{0}=\right.$ Depot, $x_{1}, . ., x_{n(\gamma)}=$ Depot $\}$, cannot appear more than CAP times. We deduce $\Sigma_{x \text { deficit }} L$ $\operatorname{DIST}(\gamma(x, u))+\Sigma_{x \text { excess }} \operatorname{L-DIST}(\gamma(x, w)) \leq C A P . L-D I S T(\gamma)$ and we conclude.

\section{A PROJECTED FLOW BASED HEURISTIC FOR NON PREEMPTIVE VSR}

We still focus here on Non Preemptive VSR problem, and derive from the LB-Proj-Flow lower bound a heuristic scheme which relies on the reconstruction, from a Projected-VSR-Flow solution, of a feasible Non Preemptive VSR solution. In order to describe it, we first introduce a feasibility oriented version of the Load-NP-VSR model:

Feasibility-Load-NP-VSR Model: \{Given a route collection $\Gamma_{\text {Route }}^{*}$, compute on the network $H\left(\Gamma_{\text {Route }}^{*}\right)$ of Section 2.2 a non negative integral arc indexed flow vector $Z$ such that:

$\circ$ for any arc-tour e, $Z_{e} \leq C A P$;

$\circ$ for any $\operatorname{arc} e=(s, \operatorname{Exc}(x)), x$ excess, $Z_{e} \leq v(x)$; for any arc $e=(\operatorname{Def}(y), p), y$ deficit, $Z_{e} \leq-v(x)$

○ Maximize $\left.Z_{p, s}\right\}$.

Then a synthetic description of our heuristic scheme comes as follows:

\section{Projected-Vehicle-Flow Algorithm}

$\Gamma_{\text {Route }}^{*} \leftarrow$ Nil

While coefficients $v(x), x \in X$ are not null do

Compute an optimal solution $(H, h)$ of the Projected-VSR-Flow model;

Derive a route collection $\gamma_{\text {Route }}^{*}=\left\{\gamma_{\text {Route }}(1), \ldots, \gamma_{\text {Route }}(P)\right\}$ from $H$;

Apply Feasibility-Load-NP-VSR to the route collection $\gamma_{\text {Route }}^{*} \cup \gamma_{\text {Route }}^{*}$ and get a resulting flow vector $Z ; \Gamma_{\text {Route }}^{*} \leftarrow \Gamma_{\text {Route }}^{*} \cup \gamma_{\text {Route }}^{*}$. Accordingly update coefficients $v(x), x \in X: v(x) \leftarrow v(x)-Z_{(s, E x c(x))}$;

Apply to the resulting route collection $\Gamma_{\text {Route }}^{*}=\left\{\Gamma_{\text {Route }}(1), \ldots, \Gamma_{\text {Route }}(K)\right\}$ the Load-NP$V S R$ algorithm, and remove from the routes $\Gamma_{\text {Route }}(k)$ all stations which do not involve any effective load/unload transaction.

We must now go further into the description of key instructions (I1) and (I2): 
- (I1): Handling of the VSR-Flow model: We do it here through the use of a MIP library, while imposing a threshold on the computing time, as soon as the number of stations exceeds 30.

- (I2): Derive a route collection $\gamma_{\text {Route }}^{*}=\left\{\gamma_{\text {Route }}(1), \ldots, \gamma_{\text {Route }}(P)\right\}$ from $H$ and $h$ : Flow vector $H$ defines a collection of $\operatorname{arcs}(x, y)$, each of them taken $H_{(x, y)}$ times, in such a way that for any node $x$, there exists as many arcs which enter into $x$ as arcs which come out $x$. So, every connected component $X_{j}, j=1 \ldots s$, of the resulting graph gives rise to some Eulerian route $\gamma_{j}$. Then we build $\gamma_{\text {Route }}^{*}$ by starting from Depot, reaching some closest $X_{j}$ into some node $x_{j}$, running $\gamma_{j}$ until being back to $x_{j}$ and keeping on with another connected component $X_{j}$. Every time the length L-DIST of current route $\gamma_{\text {Route }}(p)$ is on the edge to exceed the T-Max threshold, we close it and start $\gamma_{\text {Route }}(p+1)$.

As a matter of fact, since there exists several ways to perform this route construction process, we do it while simulating related loading/unloading transactions and trying to maximize them:

\section{Route-Reconstruction Algorithm:}

Input: the Flow vector $H$, and the $v(x), x \in X$ coefficients;

Initialization: For any $x, u(x) \leftarrow v(x) ; P \leftarrow 1$; H-Cour $\leftarrow H$; Penalty $\leftarrow 0$; Profit $\leftarrow 0$;

While $H$-Cour $\neq 0$ do

Not Stop $; x$-cour $\leftarrow$ Depot $; \gamma_{\text {Route }}(P) \leftarrow\{$ Depot, Depot $\} ;$ Load $\leftarrow 0 ;$ Length $\leftarrow 0$; While Not Stop do

1th case: There exists at least one station $y$ such that:

$$
\begin{gathered}
\text { Length }+ \text { DIST }_{x \text {-cour }}, y+D_{I S T_{y, \text { Depot }}} \leq T \text {-Max } \\
\text { and } \quad H_{x \text {-cour }}, y \neq 0
\end{gathered}
$$

For any such a station $y$, compute $L(y)=\operatorname{Inf}(C A P-\operatorname{Load}, v(y))$ in case $y$ is excess, and $L(y)=\operatorname{Inf}(\operatorname{Load},-v(y))$ in case $y$ is deficit;

Pick up $y_{0}$ which satisfies $(*)$ and $(* *)$ and is such that $L\left(y_{0}\right)$ is maximal; Move to $y_{0}: u\left(y_{0}\right) \leftarrow u\left(y_{0}\right)-L\left(y_{0}\right) ;$ Load $\leftarrow$ Load $+L\left(y_{0}\right) ; H_{x \text {-cour }, y 0} \leftarrow$ $H_{x \text {-cour }, y 0}-1 ;$ Length $\leftarrow$ Length + DIST $_{x \text {-cour, } y 0}$; Profit $\leftarrow$ Profit $+L\left(y_{0}\right)$; $x$-cour $\leftarrow y_{0}$;

2th case: 1 th case does not hold, but there exists $y$ such that $(*)$;

For any such $y$ compute $L(y)$ as above;

Pick up station $y_{0}$ which satisfies $(*)$ and is such that (bi-criteria choice):

- $L\left(y_{0}\right)$ is large and $\operatorname{COST}_{x, y 0}$ is small;

Move to $y_{0}$ as $I$ the first case with $H_{x \text {-cour } y 0}$ unchanged and Penalty $\leftarrow$ Penalty $+\operatorname{COST}_{x, y 0}$; 
3th case: None among previous cases 1 and 2 holds;

If $x$-cour is an excess station, then move back along $\gamma_{\text {Route }}(P)$ until $x$-cour is a deficit station; Close current route $\gamma_{\text {Route }}(P)$ by coming back from $x$-cour to Depot; Stop;

$$
P \leftarrow P+1
$$

Remark 8. Route-Reconstruction aims at building $\gamma_{\text {Route }}$ in such a way it maximizes Profit and minimizes both Penalty and $P$. Tree search would be too costly. Instead, we randomize Route-Reconstruction and launch it several times, before keeping the best collection $\gamma_{\text {Route }}$ ever obtained.

\section{A FLOW RECONSTRUCTION HEURISTIC FOR PREEMPTIVE VSR}

We deal now with the preemptive version of VSR, and involve the Dynamic Network Framework of Section 2.4, according to the following algorithmic scheme:

\section{Flow-Reconstruction-P-VSR Algorithm:}

1th step: Compute an optimal solution $(H, h)$ of the VSR-Flow model;

2th step: Denote by $G^{h}$ the network induced by non null $h_{x, y}$ values; Because of the optimality of $(H, h), G^{h}$ does not contain any circuit; Add 2 nodes Depot $_{1}$ and Depot 2 to $G^{h}$ and:

- Connect Depot $_{1}$ to any minimal node (which admits no predecessor but $s$ ) $x \neq s$ of $G^{h}$;

- Connect any maximal node (which admits no successor but $p$ ) $y \neq p$ of $G^{h}$ to Depot $_{2}$;

- Provide related arcs with DIST values in a natural way;

Denote by $G *^{h}$ the resulting network; flow vector $h$ may be considered as defined on $G *^{h}$;

3th step: Compute largest paths, according to DIST, respectively from Depot $_{1}$ to any node $x$ of $G *^{h}$, and from any node $y$ of to Depot $_{2}$; Denote by $L-D I S T_{x}$ and $L-D I S T^{*} y$ the resulting DIST-length values; In case $x=s$, set $L-D I S T_{s}=0$ and do as if any arc $(s, x)$ where provided with null DIST value; Do the same thing with $p$ and $L-D I S T^{*}$;

4th step: Until $L-D I S T_{\text {Depot } 2} \leq T-$ Max do Refine $G *^{h}$ and $h$;

5th step: Derive from $h$ a flow vector $f$ defined on the dynamic network $G_{T-M a x}=$ $\left(X_{T-M a x}, E_{T-M a x}\right)$ and which satisfies (E7, E11) of the Network-Flow-VSR model;

6th step: Derive a feasible solution $(F, f)$ of the Network-Flow-VSR model.

Let us now describe into more details the contents of steps 4,5 and 6 . 


\section{- Step 4: Refine procedure.}

Let us consider some node $x \neq s, p$, Depot $_{1}$, Depot $_{2}$ and such that $L-D I S T_{x}+L-$ DIST $_{x}^{*}=$ $L-D I S T_{\text {Depot2 }}$ (critical node), together with some integral number $w$ between 1 and $\left(\Sigma_{y} h_{x, y}\right)-1$. We may rank predecessors (successors) $y$ of $x$ according to increasing $L$ $D I S T_{y}+D I S T_{y, x}$ values (decreasing $L-D I S T_{y}^{*}+D I S T_{x, y}$ values). Then we define the Split procedure as follows (see Fig. 4):

\section{Procedure Split $(x, w)$ :}

Make two copies $x^{\prime}$ and $x^{\prime \prime}$ of $x$;

Assign flow values $h_{y, x^{\prime}}$ to the $\operatorname{arcs}\left(y, x^{\prime}\right), y$ predecessor of $x$, in such a way that:

$\circ$ they do not exceed $h_{y, x}$ values;

○ $\Sigma_{y} h_{y, x^{\prime}}=w$

$\circ$ the vector $h^{x^{\prime}}=\left(h_{y, x^{\prime}}, y\right.$ predecessor of $\left.x\right)$ is maximal according to the lexicographic order related to above defined ranking;

Assign remaining flow values $h_{y, x}-h_{y, x^{\prime}}, y$ predecessor of $x$, to the $\operatorname{arcs}\left(y, x^{\prime \prime}\right)$;

Do the same thing with arcs $\left(x^{\prime}, y\right)$ and $\left(x^{\prime \prime}, y\right), y$ successor of $x$, while taking into account that successors of $x$ are ranked through decreasing $L-D I S T_{y}^{*}+D I S T_{x, y}$ values;

Delete node $x$; Delete arcs $\left(y, x^{\prime}\right)$ and $\left(x^{\prime \prime}, y\right)$ which are provided with null flow values $h_{y, x^{\prime}}$, $h_{x^{\prime \prime}, y}$;

Compute:

$\circ \Delta^{\prime}=\operatorname{Sup}_{y \text { predecessor } x^{\prime}}\left(L-D I S T_{y}+D I S T_{y, x^{\prime}}\right)+\operatorname{Sup}_{y \text { successor } x^{\prime}}\left(L-D I S T_{y}^{*}+D I S T_{x^{\prime}, y}\right)$;

○ $\Delta^{\prime \prime}=\operatorname{Sup}_{y}$ predecessor $x^{\prime \prime}\left(L-D I S T_{y}+D I S T_{y, x^{\prime \prime}}\right)+\operatorname{Sup}_{y \text { successor } x^{\prime}}\left(L-D I S T_{y}^{*}+D I S T_{x^{\prime \prime}, y}\right)$;

$\circ \Delta=\operatorname{Sup} \Delta^{\prime}, \Delta^{\prime \prime}$. (N.B: $\Delta$ should be no larger than $L-D I S T_{\text {Depot } 2}$ )
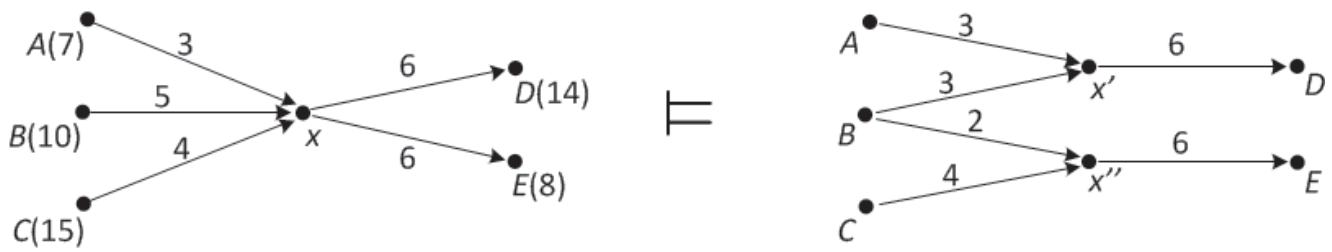

Figure 4 - The Split Mechanism.

Then the Refine procedure comes as follows:

Procedure Refine: 
Compute $x$ and $w$ in such a way that resulting $\Delta$ value be the smallest possible;

Replace current network $G^{* h}$ and related flow vector $h$ by the graph and flow vector which derive from application of $\operatorname{Split}(x, w)$;

Update $L-D I S T_{y}$ and $L-D I S T^{*}$ y values;

\section{- Step 5: Construction of flow vector $f$}

To every node $x^{*} \neq$ Depot $_{1}$, Depot $_{2}$ in the refined graph $G^{* h}$, correspond both a station $x$ and a time value $t=L-D I S T_{x}^{*}$. That means that we may associate with $x^{*}$ a node $(x, t)$ of the network $G_{T-\text { Max }}$. In case $\left(x^{*}, y^{*}\right)$ is an arc of $G^{* h}$ such that related nodes $(x, t),(y, u)$ of $G_{T-M a x}$ satisfy $u-t>D I S T_{x, y}$ then we insert a node $\left(x, u-D I S T_{x, y}\right)$, and split the $\operatorname{arc}\left(x^{*}, y^{*}\right)$ into two $\operatorname{arcs}\left((x, t),\left(x, u-D I S T_{x, y}\right)\right)$, and $\left(\left(x, u-D I S T_{x, y}\right),(y, u)\right)$. Flow vector $h$ is updated accordingly (see Fig. 5).

Once this has been done, we consider, for any station $x$, all nodes $(x, t)$ of $G_{T-M a x}$ which have been created this way, add nodes $(x, 0)$ and $(x, T$-Max), and rank all those nodes through increasing $t$ values $\left(t_{0}=0, \ldots, t_{I}=T\right.$-Max $)$. Then we replace $\operatorname{arcs}((x, t)$, $\left.\left(x, u-D I S T_{x, y},\right)\right)$ by $\operatorname{arcs}\left(\left(x, t_{i}\right),\left(x, t_{i+1}\right)\right)^{I n}$ and $\left(\left(x, t_{i}\right),\left(x, t_{i+1}\right)\right)^{\text {Out }}, i=0, \ldots, t_{I-1}$, and distribute in a natural way all flow values $h_{(x, t),(x, u)}, u>t$, previously obtained, between $\operatorname{arcs}\left(\left(x, t_{i}\right),\left(x, t_{i+1}\right)\right)^{I n}$ and $\left(\left(x, t_{i}\right),\left(x, t_{i+1}\right)\right)^{\text {Out }}$. We do it in a way which is consistent with both capacities $C A P$ and $C$, and which minimizes the sum of flow values $h$ on the $\operatorname{arcs}\left(\left(x, t_{i}\right),\left(x, t_{i+1}\right)\right)^{I n}$. Finally, we remove nodes Depot $_{1}$ and Depot $_{2}$, and assign flow values to $\operatorname{arcs}(s,(x, 0))$ and $\operatorname{arcs}((y, T-M a x), p)$ in such a way relations (E10, E11) of the Network-Flow-VSR model be satisfied. By doing this, we turn $h$ into a flow vector $f$, which may be considered as defined on an implicit representation of $G_{T-M a x}$ and which satisfies (E7, E11) of the Network-Flow-VSR model. We check that the resulting $\operatorname{cost} \Sigma_{e} \in$ ET-Maxf $_{e}$. Vehicle-Cost $t_{e}$ only differs from initial $\Sigma_{\text {arcs } e} C V_{e} . h_{e}$ by the time vehicles spend inside the carriers on arcs $\left(\left(x, t_{i}\right),\left(x, t_{i+1}\right)\right)^{I n}$.
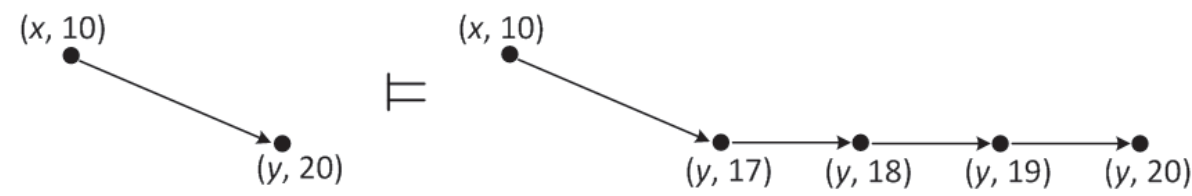

Figure 5 - Decomposing an arc $((x, 10),(y, 20))$ such that $\operatorname{DIST}_{x, y}=7$.

\section{- Step 6: Construction of flow vector $F$}

We complete the construction of step 5 by introducing nodes (Depot, 0), (Depot, T-Max) and all nodes (Depot, $t)$ such that there exists $(x, u)$, obtained through step 5 and such that $u=t+D I S T_{\text {Depot }, x}$ or $t=u+D I S T_{x, \text { Depot }}$. We rank those nodes according to increasing $t$ values $\left(t_{0}=0, . ., t_{S}=T\right.$-Max $)$, and connect them with arcs $\left(\left(\text { Depot }, t_{i}\right),\left(\text { Depot }, t_{i+1}\right)\right)^{I n}$ 
and $\left(\left(\text { Depot }, t_{i}\right),\left(\text { Depot }, t_{i+1}\right)\right)^{\text {Out }}$ accordingly. We also connect any node (Depot, $\left.t\right)$ obtained this way to any existing node $(x, u)$ such that $u=t+D I S T_{\text {Depot, } x}$ and any existing node $(x, u)$ to node $($ Depot,$t)$ such that $t=u+D I S T_{x, \text { Depot }}$. We may consider the resulting network $G^{* f}$ as a sub-network of $G_{T-\text { Max }}$, and its arcs $e$ as provided with carrier costs $C C_{e}$ as in the VSR-Flow-Model. Then we compute on $G^{* f}$ a flow vector $F$, which satisfies (E7, E8, E9, E10) and which minimizes $\Sigma_{e \in E T-M a x} F_{e}$. Carrier-Cost $e_{e}$.

Remark 9. Step 6 always yields a feasible solution, since no non null vehicle flow value $f_{(x, t),(y, u)}, x \neq y$, is involved with $t<D I S T_{\text {Depot }, x}$ or $(T-M a x-u)<D I S T_{y, \text { Depot. }}$.

\section{NUMERICAL EXPERIMENTS}

Purpose: Our purpose here is to:

- get a comparative evaluation of the lower bounds of Section 3;

- get a comparative evaluation of the 3 heuristic scheme described in Section 4, 5 and 6.

- test the influence of scaling coefficients $\alpha, \beta, \delta$ and the impact of preemption.

Technical context: Algorithms were implemented in C, on PC AMD Opteron 2.1GHz, while using gec 4.1 compiler. We used the CPLEX12 library for the handling of linear models.

Instances: No standardized benchmarks exist for generic VSR. So we built instances as follows:

- Station set $X$ is randomly generated as a set of $n+1$ points $x_{0}, x_{1}, \ldots, x_{n}$, inside the $[0,10] \times[0,10]$ sub-square of the Euclidean $2 D$-space;

- DIST corresponds to the Euclidean Distance; COST corresponds either to a multiple of either the Euclidean distance or the Sum distance DIST-S $(x, y),\left(x^{\prime}, y^{\prime}\right)=\left|x^{\prime}-x\right|+\mid y^{\prime}-$ $y \mid: C O S T=\lambda . D I S T$ or $C O S T=\lambda . D I S T-S$;

- Each station but Depot $=x_{0}$ is assigned a random $v(x)$ value chosen between -10 and 10 , in such a way that the sum of demands over all stations equal to 0 ; That means that we allow here few neutral stations.

- CAP is randomly chosen between 10 and 20;

- T-Max is randomly chosen between $=30$ and 100 .

\subsection{Testing the Impact of Scaling Coefficients $\alpha, \beta, \delta$}

On a given instance (X,v,CAP, T-Max, DIST, COST $=D I S T)$, we fix $\alpha=10$, make vary $\beta, \delta$ with $\beta+\delta=1$, and compute solutions through the Shortest Cost/Distance Strategy. We obtain 


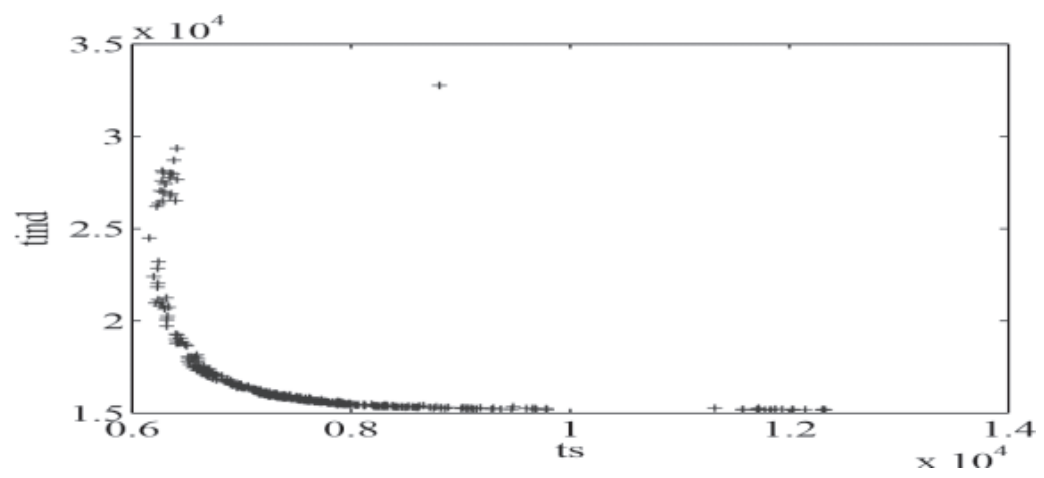

Figure 6 - Pareto frontier carrier riding cost versus vehicle riding time.

the Pareto frontier of Figure 6, with $t$ d denoting the carrier riding cost and tind the vehicle riding time.

Comment: We see that Carrier riding cost and vehicle riding time behave like antagonistic criteria.

\subsection{Comparing the Lower Bounds of Section 3}

For several groups of 5 instances each related to a given size $n$, we compute the mean value of:

- LB-Proj-Flow: as defined in Theorem 5; CPU-LB-Proj denotes the related computing times in seconds.

- $L B-M C A$ as defined in Theorem 3; $C P U-L B-M C A$ denotes the related computing times in seconds.

When $n$ is larger than 35, computing times for LB-Proj-Flow are too high. Still, we observe that CPLEX12 converges fast on the LB-Proj-Flow model, and so that imposing a threshold on CPU time is not likely to deteriorate the $L B$-Proj-Flow value in a significant way, even if it keeps us from mathematically proving that we get a lower bound this way. We get the following results (Symbol* means that we imposed a threshold of $1000 \mathrm{~s}$ on the running time for the LB-Proj-Flow model):

Comment: Experiments confirm both the better quality and the higher computing cost of the LB-Proj-Flow Lower bound.

\subsection{Testing the Heuristics of Section 4, 5, 6 and the impact of Preemption}

We compute, for the same groups of 5 instances as above, the average of the following GlobalCost values: 
Table 1 - Lower Bounds with $\alpha=10, \beta=1, \delta=0$.

\begin{tabular}{c|c|c|c|c}
\hline$n$ & LB-Proj-Flow & CPU-LB-Proj & LB-MCA & CPU-LB-MCA \\
\hline 20 & 84.8 & 46.6 & 82.3 & 8.6 \\
\hline 30 & 96.5 & 3204.1 & 84.6 & 28.5 \\
\hline 40 & 108.4 & $1000^{*}$ & 92.2 & 50.4 \\
\hline 50 & 135.1 & $1000^{*}$ & 117.8 & 70.3 \\
\hline 60 & 141.5 & $1000^{*}$ & 130.1 & 99.1 \\
\hline
\end{tabular}

Table 2 - Lower Bounds with $\alpha=10, \beta=0, \delta=1$.

\begin{tabular}{c|c|c|c|c}
\hline$n$ & LB-Proj-Flow & CPU-LB-Proj & LB-MCA & CPU-LB-MCA \\
\hline 20 & 182.7 & 67.6 & 176.9 & 6.5 \\
\hline 30 & 228.2 & $1000^{*}$ & 216.2 & 25.7 \\
\hline 40 & 235.6 & $1000^{*}$ & 218.7 & 52.6 \\
\hline 50 & 299.9 & $1000^{*}$ & 288.3 & 75.4 \\
\hline 60 & 297.3 & $1000^{*}$ & 270.1 & 109.0 \\
\hline
\end{tabular}

- SD: obtained through Shortest Cost/Distance Strategy initialization of VSR-MCA $\Rightarrow C P U$ $S D$ is the related CPU time (s).

- $S D(50)$ : obtained through GRASP-VSR-MCA, with $N=1$ and $R=50$.

- $L S(50)$ : obtained through through GRASP-VSR-MCA, with $N=50$ and $R=1 \Rightarrow C P U$ $L S$ is the related CPU time.

- VF: obtained through the Projected-Vehicle-Flow heuristic $\Rightarrow C P U-V F$ is the related CPU time.

- FRP: obtained through application of the Flow-Reconstruction-P-VSR algorithm $\Rightarrow C P U$ $F R P$ is the related CPU time.

- $L B$ denotes here the LB-Proj-Flow lower bound of the previous experiment.

We get (the computing time which were necessary in order to deal with the LB-Proj-Flow model re not taken into account in $C P U-V F$ and $C P U-F R P)$ :

Comment: The improvement margin induced by the local search loop of the VSR-MCA algorithm is not very high, especially when the focus is on the vehicle riding time. A consequence is that performing random diversification through the use of the replication parameter $R$ is most often more efficient. Both require small computational times. The Projected-Vehicle Flow algorithm provides similar results. At the end, the Flow-Reconstruction-P-VSR algorithm produces preemptive solutions whose Global-Cost value is always better that values obtained for the Non 
Table 3 - Values $S D, S D(50), L S(50), V F, F R P$ with $\alpha=10, \beta=1, \delta=0$.

\begin{tabular}{c|c|c|c|c|c|c|c|c|c|c}
\hline$n$ & $L B$ & $S D$ & $C P U-S D$ & $S D(50)$ & $L S(50)$ & $C P U-P I$ & $V F$ & $C P U-V F$ & $F R P$ & $C P U-F R P$ \\
\hline 20 & 84.8 & 99.5 & 0.3 & 94.7 & 96.3 & 1.1 & 92.3 & 4.7 & 88.5 & 3.6 \\
\hline 30 & 96.5 & 120.5 & 0.7 & 113.6 & 112.5 & 2.9 & 108.9 & 9.6 & 103.6 & 38.5 \\
\hline 40 & 108.4 & 152.6 & 1.2 & 136.1 & 139.7 & 5.4 & 132.0 & 14.1 & 119.5 & 70.2 \\
\hline 50 & 135.1 & 182.3 & 1.5 & 169.0 & 164.0 & 8.7 & 161.8 & 19.3 & 146.7 & 95.6 \\
\hline 60 & 141.5 & 200.1 & 1.8 & 178.5 & 176.7 & 12.0 & 169.3 & 25.5 & 150.3 & 119.3 \\
\hline
\end{tabular}

Table 4 - Values $S D, S D(50), L S(50), V F, F R P$ with $\alpha=10, \beta=0, \delta=1$.

\begin{tabular}{c|c|c|c|c|c|c|c|c|c|c}
\hline$n$ & $L B$ & $S D$ & $C P U-S D$ & $S D(50)$ & $L S(50)$ & $C P U-P I$ & $V F$ & $C P U-V F$ & $F R P$ & $C P U-F R P$ \\
\hline 20 & 182.7 & 220.0 & 0.4 & 212.1 & 217.6 & 1.4 & 205.6 & 5.8 & 191.0 & 3.9 \\
\hline 30 & 228.2 & 273.1 & 0.8 & 264.6 & 270.9 & 3.3 & 255.7 & 10.2 & 241.5 & 33.5 \\
\hline 40 & 235.6 & 297.5 & 1.3 & 277.7 & 288.7 & 6.0 & 264.6 & 14.9 & 247.3 & 64.0 \\
\hline 50 & 299.9 & 372.2 & 1.5 & 346.3 & 364.7 & 9.5 & 335.9 & 21.0 & 312.0 & 120.3 \\
\hline 60 & 297.3 & 378.4 & 1.9 & 348.9 & 369.8 & 11.8 & 340.8 & 28.4 & 321.9 & 122.7 \\
\hline
\end{tabular}

Preemptive case. This should be amplified in case we allow more neutral stations. We may extrapolate that, on our instances, lower bound LB-Proj-Flow probably misses the optimal value of Non Preemptive VSR problem by about $10 \%$, and is close to optimal value of Preemptive VSR. Finally, we must notice that a limitation for both Flow-Reconstruction-P-VSR and ProjectedVehicle-Flow is that they rely on the resolution of Projected-VSR-Flow instances defined on an almost complete oriented graph, whose computing costs increase fast with the number $n$ of stations.

\section{CONCLUSION}

We mainly dealt here with a Vehicle Sharing Relocation problem, related to the operational management of Vehicle Sharing systems, and which we handled according to Network Flow approaches which puts the focus on the way vehicles move from excess stations to deficit ones. Still, many open problems remain, related to the design of exact algorithms and also, if we refer to practical context, to the way algorithms which have been designed for static models may be adapted in order to fit with on line contexts. Future research will be carried on in order to address these issues.

\section{REFERENCES}

[1] Anily S, Gendreau M \& Laporte G. 2011. The uncapacitated swapping problem on line and circle. Networks, 58: 83-94.

[2] Archetti C \& Speranza M. 2008. The split delivery vehicle routing problem, a survey; in The vehicle routing problem: latest adances and new challenges; p. 103-122, Springer U.S. 
[3] ARONSOn J. 1989. A survey on dynamic network flows. Annals of O.R, 20: 1-66.

[4] BARTH M \& TODD M. 1999. Simulation model performance analysis of a multiple station shared vehicle system. Transportation Research Part C: Emerging Technologies, 7(4): 237-259.

[5] Benchimol M de P, Chappert B, Taille a De la, Laroche F, Meunier F \& Robinet L. 2011. Balancing the stations of a self service bike hiring systems. RAIRO-RO, 45: 37-61.

[6] Bordenave C, Gendreau M \& Laporte G. 2012. A branch and cut algorithm for the preemptive swapping problem. Networks, 59(4): 387-399.

[7] Boyaci B, Zografos K \& Geroliminis N. 2015. An optimization framework for the development of efficient one-way car-sharing systems. EJOR, 240(3): 718-733.

[8] Carlier A, Munier-Kordon A \& Klaudel W. 2015. Mathematical model for the study of relocation strategies in one-way carsharing systems. Transportation Research Procedia, 10: 374 383.

[9] CARLIER A. 2016. PhD PARIS VI University, France.

[10] Chemla D, Meunier F \& Calvo RW. 2013. Bike sharing systems: solving the static rebalancing problem. Discrete Optimization, 10(2): 120-146.

[11] Contardo C, Morency C \& Rousseau LM. 2012. Balancing a dynamic public bike-sharing system. Rapport CIRRELT, Univ. MONTREAL.

[12] Cordeau JF \& Laporte G. 2003. A Tabu search heuristic algorithm for the static multi-vehicle Dial and Ride Problem. Transportation Research B, 37: 579-594.

[13] Cornuejols G, Nemhauser GL \& Wolsey LA. 1990. "The uncapacited facility location problem”. In: Mirchandani PB \& Francis RL (Eds.), Discrete Location Theory, p. 119-171.

[14] Gavalas D, Konstantopoulos C \& Pantziou G. 2015. Design and management of vehicle sharing systems: a survey of algorithmic approaches; ArXiv e-prints, arxiv.org/pdf/1510.01158, October.

[15] Ghiani G, Guerrero F \& Musmanno F. 2002. "The capacitated plant location problem with multiple facilities in the same site”. Computer and Operations Research, 29-13: 1903-1912.

[16] Hansen P, Brimberg J, Urosevic D \& Mladenovic N. 2007. "Primal-dual local search for plant location". INFORMS Jour. Comp., 19-4: 58-72.

[17] Perez HH \& GonZalez JS. 2004. Heuristics for the one commodity pick up and delivery traveling salesman problem. Transportation Sciences, 38: 244-255.

[18] Kek GH, Cheu RL, Meng Q \& Fung C.HA. 2009. A decision support system for vehicle relocation operations in carsharing. Transport. Res. E: Logistics/Transp. Review, 45(1): 149-158.

[19] Klose A \& DreXl A. 2005. "Facility location models for distribution systems". EJOR, 162: 429449.

[20] LeE J \& PARK GL. 2013. Design of a team based relocation scheme in electric vehicle sharing systems. Proc. Int. Conf. Computational Sciences and Applications, 7973: 368-377.

[21] Lee J, Park GL, Kang MJ, Kim J, Kim HJ, Kim LK \& Ko YI. 2012. Design of an efficient matching based relocation scheme for electric vehicle sharing systems. Proc. Int. Conf. in Computer Applications for Modeling, Simulation and Automobile, 341: 109-115. 
[22] Mirchandani PB \& Francis RL. 1990. Discrete Location Theory. J. Wiley Sons.

[23] NourinedJAD M \& Roorda MJ. 2014. A dynamic car sharing decision support system. Transportation Research E, 66: 36-50.

[24] Rainer-Harbach M, PAPAZECK P, Hu B \& RaidL G. 2015. Balancing bicycle sharing systems: a variable neighbourhood search approach. Journal of Global optimization, 63(3): 597-629.

[25] Raviv T, Tzur M \& Forma I. 2013. Static repositioning in a bike sharing system: models and solution approaches. EURO Journal on Transportation and Logistics, 2(3): 187-229.

[26] Resende M \& WeRneck R. 2004. "Hybrid heuristic for p-median problem". J. Heuristics, 10: $59-88$.

[27] Sayarshad H, TAVAssoli S \& Zhao F. 2012. A multi-periodic optimization formulation for bike planning and bike utilization. Applied Mathematical Modelling, 36(10): 4944-4951.

[28] Shaheen SA, Guzman S \& Zhang H. 2010. Bike sharing in Europe, the Americas and Asia. Transportation Res. Record: Journal Transportation Research Board, 2143(1): 159-167.

[29] Waserhole A, Jost V \& Brauner N. 2012. Vehicle sharing system pricing regulation, http://hal.archivesouvertes.fr/docs/00/82/81/92/PDF/.

[30] WHITAKER R. 1983. "A fast algorithm for the greedy interchange of large scale clustering and median location problems". INFOR, 21: 95-108. 